\title{
On the symmetry function of a convex set
}

\author{
Alexandre Belloni • Robert M. Freund
}

Received: 21 July 2004 / Accepted: 1 October 2005 / Published online: 12 December 2006 (C) Springer-Verlag 2006

Abstract We attempt a broad exploration of properties and connections between the symmetry function of a convex set $S \subset \mathbb{R}^{n}$ and other arenas of convexity including convex functions, convex geometry, probability theory on convex sets, and computational complexity. Given a point $x \in S$, let $\operatorname{sym}(x, S)$ denote the symmetry value of $x$ in $S$ :

$$
\operatorname{sym}(x, S):=\max \{\alpha \geq 0: x+\alpha(x-y) \in S \text { for every } y \in S\},
$$

which essentially measures how symmetric $S$ is about the point $x$, and define

$$
\operatorname{sym}(S):=\max _{x \in S} \operatorname{sym}(x, S)
$$

$x^{*}$ is called a symmetry point of $S$ if $x^{*}$ achieves the above maximum. The set $S$ is a symmetric set if $\operatorname{sym}(S)=1$. There are many important properties of symmetric convex sets; herein we explore how these properties extend as a function of $\operatorname{sym}(S)$ and/or $\operatorname{sym}(x, S)$. By accounting for the role of the symmetry function, we reduce the dependence of many mathematical results on the strong assumption that $S$ is symmetric, and we are able to capture and otherwise

Dedicated to Clovis Gonzaga on the occasion of his 60th birthday.

A. Belloni $(\bowtie)$

IBM T. J. Watson Research Center and MIT, 32-221, 1101 Kitchawan Road,

Yorktown Heights, NY 10598 USA

e-mail: belloni@mit.edu

R. M. Freund

MIT Sloan School of Management, 50 Memorial Drive, Cambridge, MA 02139-4307, USA

e-mail: rfreund@mit.edu 
quantify many of the ways that the symmetry function influences properties of convex sets and functions. The results in this paper include functional properties of $\operatorname{sym}(x, S)$, relations with several convex geometry quantities such as volume, distance, and cross-ratio distance, as well as set approximation results, including a refinement of the Löwner-John rounding theorems, and applications of symmetry to probability theory on convex sets. We provide a characterization of symmetry points $x^{*}$ for general convex sets. Finally, in the polyhedral case, we show how to efficiently compute $\mathbf{s y m}(S)$ and a symmetry point $x^{*}$ using linear programming. The paper also contains discussions of open questions as well as unproved conjectures regarding the symmetry function and its connection to other areas of convexity theory.

Mathematics Subject Classification (2000) $90 \mathrm{C} 25 \cdot 65 \mathrm{~K} 05 \cdot 90 \mathrm{C} 27$

\section{Introduction}

We attempt a broad exploration of properties and connections between the symmetry function of a convex set $S \subset \mathbb{R}^{n}$ and other areas of convexity including convex functions, convex geometry, probability theory on convex sets, and computational complexity. Given a closed convex set $S$ and a point $x \in S$, define the symmetry of $S$ about $x$ as follows:

$$
\operatorname{sym}(x, S):=\max \{\alpha \geq 0: x+\alpha(x-y) \in S \text { for every } y \in S\},
$$

which intuitively states that $\operatorname{sym}(x, S)$ is the largest scalar $\alpha$ such that every point $y \in S$ can be reflected through $x$ by the factor $\alpha$ and still lie in $S$. The symmetry value of $S$ then is:

$$
\operatorname{sym}(S):=\max _{x \in S} \operatorname{sym}(x, S),
$$

and $x^{*}$ is a symmetry point of $S$ if $x^{*}$ achieves the above maximum (also called a "critical point" in [12], [14] and [18]). $S$ is symmetric if $\mathbf{s y m}(S)=1$. There are a variety of other measures of symmetry (or asymmetry) for a convex set that have been studied over the years, see Grünbaum [12] for example; the symmetry measure based on (2) is due to Minkowski [18], which in all likelihood was the first and most useful such symmetry measure.

We explore fundamental properties of $\operatorname{sym}(x, S)$, and we present new results in other areas of convexity theory that are connected to the symmetry function. In Sect. 2 we examine functional properties of $\operatorname{sym}(x, S)$. We show that $\operatorname{sym}(x, S)$ is a quasiconcave function, and more importantly, that $\operatorname{sym}(x, S)$ is a $\log c o n-$ cave function and therefore inherits some of the strong results of logconcave functions related to sampling on convex sets (Theorem 1). We also show that $\operatorname{sym}(x, S)$ is the infimum of linear fractional functions related to the supporting hyperplanes of $S$ (Proposition 1). In Proposition 3 we explore the behavior of $\operatorname{sym}(x, S)$ under basic set operations such as intersection, Minkowski sums, polarity, Cartesian product, and affine transformation. And in Proposition 2 we characterize $\operatorname{sym}(x, S)$ when $S$ is symmetric. 
In Sect. 3 we focus on connections between $\operatorname{sym}(x, S)$ and a wide variety of geometric properties of convex bodies, including volume ratios, distance metrics, set-approximation and rounding results, and probability theory on convex sets. It is well-known that any half-space whose bounding hyperplane passes through the center of mass $z_{S}$ of $S$ will cut off at least $1 / \mathbf{e}$ and at most $1-1 / \mathbf{e}$ of the volume of $S$, see Grünbaum [11]. In a similar vein, in Sect. 3.1 we present lower and upper bounds on ratios of volumes of $S$ to the intersection of $S$ with a half-space whose bounding hyperplane passes through $x$, as a function of $\operatorname{sym}(x, S)$ (Theorem 2), as well as lower bounds on the $(n-1)$-dimensional volume ratios of slices of $S$ defined by the intersection of $S$ with a hyperplane passing through $x$, as a function of $\operatorname{sym}(x, S)$ (Theorem 3).

If $S$ is a symmetric convex body, then it is a straightforward exercise to show that the symmetry point of $S$ is unique. Furthermore, if $S$ is nearly symmetric, intuition suggests that two points in $S$ with high symmetry values cannot be too far apart. This intuition is quantified Sect. 3.2, where we present upper bounds on the relative distance (in any norm) between two points $x, y \in S$ as a function of $\operatorname{sym}(x, S)$ and $\operatorname{sym}(y, S)$ (Theorem 4) and upper bounds on the "cross-ratio distance" in Theorem 5.

Section 3.3 examines the approximation of the convex set $S$ by another convex set $P$. We say that $P$ is a $\beta$-approximation of $S$ if there exists a point $x \in S$ such that $\beta P \subset S-x \subset P$. In the case when $P$ is an ellipsoid centered at the origin, then the statement " $P$ is a $\beta$-approximation of $S$ " is equivalent to " $\beta P$ provides a $\frac{1}{\beta}$-rounding of $S$." We examine the interrelationship between the symmetry function and bounds on $\beta$-approximations for $S$. We show that for any $x \in S$ there exists a $\sqrt{n} / \operatorname{sym}(x, S)$-rounding of $S$ centered at $x$ (Theorem 7). A classical example of $\beta$-approximation is given by the Löwner-John theorem [15], which guarantees a $1 / \sqrt{n}$-approximation for a symmetric convex body and a $1 / n$-approximation for general convex body using ellipsoids. Unfortunately, the theorem does not provide more precise bounds for case when $S$ is nearly symmetric, i.e., $\operatorname{sym}(S)=1-\varepsilon$ for $\varepsilon$ small. This is partially rectified herein, where we prove a slightly stronger rounding results using $\mathbf{s y m}(x, S)$ (Theorem $9)$. We also show that if two convex sets are nearly the same, then their symmetry must be nearly the same (Theorem 8 ), and we show how to construct a norm based on $\operatorname{sym}(S)$ that yields the optimal $\beta$-approximation of $S$ among all symmetric convex bodies (Lemma 1).

Section 3.4 is concerned with connections between symmetry and probability theory on convex sets. Let $X$ be a random vector uniformly distributed on $S$. We show that the expected value of $\operatorname{sym}(X, S)$ is nicely bounded from below (by $\operatorname{sym}(S) /(2(n+1)))$ and we present lower bounds on the probability that $\operatorname{sym}(X, S)$ is within a constant $M$ of $\operatorname{sym}(S)$. Furthermore, in the case when $S$ is symmetric, these quantities have closed-form expressions independent of the specific set $S$ (Theorem 10). We also present an extension of Anderson's Lemma [1] concerning the the integral of a nonnegative logconcave even function on $S$, to the case of non-symmetric convex sets (Theorem 11), which has many statistical applications. 
Since symmetry points enjoy many interesting properties, it is natural to explore methods for computing a symmetry point and for computing $\mathbf{s y m}(S)$, which is the subject of Sect. 5. As expected, the representation of $S$ plays a major role in any computational scheme. While the problem of simply evaluating the $\operatorname{sym}(x, S)$ for a given $x \in S$ is a hard problem in general, it turns out that for polyhedra, whose most common representations are as the convex hull of points and as the intersection of half-spaces, computing a symmetry point can be accomplished via linear programming. When $S$ is given as the convex hull of $m$ points, we show that determining a symmetry point can be computed by solving a linear program in $m^{2}$ nonnegative variables, or as non-differentiable concave maximization problem where subgradients can be computed by solving $m$ decoupled linear programming subproblems with only $m$ nonnegative variables each. The more interesting case is when $S$ is given as the intersection of $m$ half-spaces. Then a symmetry point and $\mathbf{s y m}(S)$ can be computed by solving $m+1$ linear programs with $m$ nonnegative variables. We present an interiorpoint algorithm that, given an approximate analytic center $x^{a}$ of $S$, will compute an approximation of $\mathbf{s y m}(S)$ to any given relative tolerance $\varepsilon$ in no more than

$$
\left\lceil 10 m^{1.5} \ln \left(\frac{10 m}{\epsilon}\right)\right\rceil
$$

iterations of Newton's method.

The paper also contains a variety of discussions of open questions as well as unproved conjectures regarding the symmetry function and its connection to other areas of convexity theory.

Notation. Let $S \subset \mathbb{R}^{n}$ denote a convex set and let $\langle\cdot, \cdot\rangle$ denote the conventional inner product in the appropriate Euclidean space. int $S$ denotes the interior of $S$. Using traditional convex analysis notation, we let aff $(S)$ be the minimal affine subspace that contains $S$ and let $S^{\perp}$ be its orthogonal subspace complement. The polar of $S$ is defined as $S^{\circ}=\left\{y \in \mathbb{R}^{n}:\langle x, y\rangle \leq 1\right.$ for all $\left.x \in S\right\}$. Given a convex function $f(\cdot)$, for $x \in \operatorname{dom} f(\cdot)$ the subdifferential of $f(\cdot)$ is defined as $\partial f(x):=\left\{s \in \mathbb{R}^{n}: f(y) \geq f(x)+\langle s, y-x\rangle\right.$ for all $\left.y \in \operatorname{dom} f(\cdot)\right\}$. Let $e=(1, \ldots, 1)^{T}$ denote the vector of ones whose dimension is dictated by context, let e denote the base of the natural logarithm, and let $\operatorname{dist}(x, T):=\min _{y \in T}\|y-x\|$ be the distance from $x$ to the set $T$ in the norm $\|\cdot\|$ dictated by context.

\section{Functional properties of $\operatorname{sym}(x, S)$}

We make the following assumption:

Assumption A $S$ is a convex body, i.e., $S$ is a nonempty closed bounded convex set with a nonempty interior.

When $S$ is a convex set but is either not closed or is unbounded, then certain properties of $\mathbf{s y m}(S)$ break down; we refer the interested reader to Appendix A for a discussion of these general cases. We assume that $S$ has an interior as a 
matter of convenience, as one can always work with the affine hull of $S$ or its subspace translation with no loss of generality, but at considerable notational and expositional expense.

There are other definitions of $\operatorname{sym}(x, S)$ equivalent to (1). In [19], $\operatorname{sym}(x, S)$ is defined by considering the set $\mathcal{L}(x, S)$ of all chords of $S$ that pass through $x$. For $L \in \mathcal{L}(x, S)$, let $r(L)$ denote the ratio of the length of the smaller to the larger of the two intervals in $L \cap(S \backslash\{x\})$, and define

$$
\operatorname{sym}(x, S)=\inf _{L \in \mathcal{L}(x, S)} r(L)
$$

Herein it will be convenient to also use the following set-containment definition of $\operatorname{sym}(x, S)$ :

$$
\operatorname{sym}(x, S)=\max \{\alpha \geq 0: \alpha(x-S) \subseteq(S-x)\}
$$

It turns out that this definition is particularly useful to motivate and prove many of our results.

Intuition suggests that $\operatorname{sym}(x, S)$ inherits many nice properties from the convexity of $S$, as our first result shows:

Theorem 1 Under Assumption A,

(i) $\operatorname{sym}(\cdot, S): S \rightarrow[0,1]$ is a continuous quasiconcave function,

(ii) $h(x, S):=\frac{\operatorname{sym}(x, S)}{1+\operatorname{sym}(x, S)}$ is a concave function on $S$, and

(iii) $\operatorname{sym}(\cdot, S)$ is a logconcave function on $S$.

Regarding part (iii) of the theorem, note that logconcave functions play a central role in the theory of probability and sampling on convex bodies, see [17]. The proof of this theorem will use the following proposition, which will also be useful in the development of an algorithm for computing $\operatorname{sym}(S)$ in Sect. 5.

Proposition 1 Let $S$ be a convex body, and consider the representation of $S$ as the intersection of halfspaces: $S=\left\{x \in \mathbb{R}^{n}: a_{i}^{T} x \leq b_{i}, i \in I\right\}$ for some (possibly infinity) index set $I$, and let $\delta_{i}^{*}:=\max _{x \in S}\left\{-a_{i}^{T} x\right\}$ for $i \in I$. Then for all $x \in S$,

$$
\operatorname{sym}(x, S)=\inf _{i \in I}\left\{\frac{b_{i}-a_{i}^{T} x}{\delta_{i}^{*}+a_{i}^{T} x}\right\} .
$$

Proof Let $\alpha=\operatorname{sym}(x, S)$ and $\gamma:=\min _{i \in I}\left\{\frac{b_{i}-a_{i}^{T} x}{\delta_{i}^{*}+a_{i}^{T} x}\right\}$. Then for all $y \in S, x+$ $\alpha(x-y) \in S$, so

$$
a_{i}^{T} x+\alpha a_{i}^{T} x+\alpha\left(-a_{i}^{T} y\right) \leq b_{i}, \quad i \in I
$$


This implies that

$$
a_{i}^{T} x+\alpha a_{i}^{T} x+\alpha \delta_{i}^{*} \leq b_{i}, i \quad \in I
$$

whereby $\alpha \leq \gamma$. On the other hand, for all $y \in S$ we have:

$$
b_{i}-a_{i}^{T} x \geq \gamma\left(\delta_{i}^{*}+a_{i}^{T} x\right) \geq \gamma\left(-a_{i}^{T} y+a_{i}^{T} x\right) .
$$

Thus $a_{i}^{T} x+\gamma a_{i}^{T} x+\gamma\left(-a_{i}^{T} y\right) \leq b_{i}$, and therefore $a_{i}^{T}(x+\gamma(x-y)) \leq b_{i}$ which implies that $\alpha \geq \gamma$. Thus $\alpha=\gamma$.

Proof of Theorem 1 We first prove (ii). It follows from Proposition 1 that

$$
h(x, S)=\frac{\min _{i \in I}\left\{\frac{b_{i}-a_{i}^{T} x}{\delta_{i}^{*}+a_{i}^{T} x}\right\}}{1+\min _{i \in I}\left\{\frac{b_{i}-a_{i}^{T} x}{\delta_{i}^{*}+a_{i}^{T} x}\right\}}=\min _{i \in I}\left\{\frac{\frac{b_{i}-a_{i}^{T} x}{\delta_{i}^{*}+a_{i}^{T} x}}{1+\frac{b_{i}-a_{i}^{T} x}{\delta_{i}^{*}+a_{i}^{T} x}}\right\}=\min _{i \in I}\left\{\frac{b_{i}-a_{i}^{T} x}{b_{i}+\delta_{i}^{*}}\right\},
$$

which is the minimum of linear functions and so is concave.

To prove (i), first observe that $\operatorname{sym}(x, S)$ is monotone in the concave function $h(x, S)$, and so is quasiconcave. To prove the continuity of $\operatorname{sym}(x, S)$ it suffices to prove the continuity of $h(x, S)$. It follows from concavity that $h(x, S)$ is continuous on int $S$. For $\bar{x} \in \partial S$ it follows from (1) that $\operatorname{sym}(x, S)=0$ and hence $h(x, S)=0$. Because $S$ is a convex body there exists a ball of radius $r>0$ that is contained in $S$. Now suppose that $x^{j} \rightarrow \bar{x}$, whereby $\operatorname{dist}\left(x^{j}, \partial S\right) \rightarrow 0$. It follows from (4) that $\operatorname{sym}\left(x^{j}, S\right) \cdot r \leq \operatorname{dist}\left(x^{j}, \partial S\right)$, whereby $\operatorname{sym}\left(x^{j}, S\right) \rightarrow 0=\operatorname{sym}(\bar{x}, S)$, showing continuity of $h(x, S)$ and hence of $\operatorname{sym}(x, S)$ on $S$.

To prove (iii) define the following functions:

$$
f(t)=\frac{t}{1+t} \quad \text { and } \quad g(t)=\ln \left(\frac{t}{1-t}\right) .
$$

For these functions, we have the following properties:

(i) $f$ is monotone, concave and $f(\operatorname{sym}(x, S)) \in[0,1 / 2]$ for any $x \in S$;

(ii) $g$ is monotone for $t \in(0,1)$ and concave for $t \in(0,1 / 2]$;

(iii) $g(f(t))=\ln t$.

Now, for any $\alpha \in[0,1], x, y \in S$,

$$
\begin{aligned}
\ln (\operatorname{sym}(\alpha x+(1-\alpha) y, S)) & =g(f(\operatorname{sym}(\alpha x+(1-\alpha) y, S))) \\
& \geq g(\alpha f(\operatorname{sym}(x, S))+(1-\alpha) f(\operatorname{sym}(y, S))) \\
& \geq \alpha g(f(\operatorname{sym}(x, S)))+(1-\alpha) g(f(\operatorname{sym}(y, S))) \\
& =\alpha \ln \operatorname{sym}(x, S)+(1-\alpha) \ln \operatorname{sym}(y, S),
\end{aligned}
$$


where the first inequality follows from the concavity of $h(\cdot, S)=f(\operatorname{sym}(\cdot, S))$ and the monotonicity of $g$, and the second inequality follows from the concavity of $g$ on $[0,1 / 2]$.

It is curious that $\operatorname{sym}(\cdot, S)$ is not a concave function. To see this, consider $S=[0,1] \subset \mathbb{R}$; then a trivial computation yields $\operatorname{sym}(x, S)=\min \left\{\frac{x}{(1-x)} ; \frac{(1-x)}{x}\right\}$, which is not concave on $S$ and is not differentiable at $x=\frac{1}{2}$. Part (ii) of Theorem 1 shows that a simple nonlinear transformation of the symmetry function is concave.

For a symmetric convex body $S$, i.e., $\operatorname{sym}(S)=1$, it is possible to prove a stronger statement and completely characterize the symmetry function using the norm induced by $S$. Suppose $S$ is a symmetric convex set centered at the origin. Let $\|\cdot\|_{S}$ denote the norm induced by $S$, namely $\|x\|_{S}:=\min \{\gamma: x \in \gamma S\}$.

Proposition 2 Under Assumption A, let $S$ be symmetric and centered at the origin. Then for every $x \in S$,

$$
\operatorname{sym}(x, S)=\frac{1-\|x\|_{S}}{1+\|x\|_{S}} .
$$

Proof We start by observing that for any $y \in S,\|y\|_{S} \leq 1$. For any $x \in S$, consider any chord of $S$ that intersects $x$, and let $p, q$ be the endpoints of this chord. Notice that $\|p\|_{S}=\|q\|_{S}=1$ and using the triangle inequality,

$$
\|p-x\|_{S} \leq\|x\|_{S}+\|p\|_{S} \quad \text { and } \quad\|q\|_{S} \leq\|q-x\|_{S}+\|x\|_{S}
$$

Thus,

$$
\frac{\|q-x\|_{S}}{\|p-x\|_{S}} \geq \frac{\|q\|_{S}-\|x\|_{S}}{\|x\|_{S}+\|p\|_{S}}=\frac{1-\|x\|_{S}}{1+\|x\|_{S}} .
$$

Finally, the lower bound is achieved by the chord that passes through $x$ and the origin.

The next proposition presents properties of the symmetry function under basic set operations on $S$.

Proposition 3 Let $S, T \subset \mathbb{R}^{n}$ be convex bodies, and let $x \in S$ and $y \in T$. Then:

1. (Superminimality under intersection) If $x \in S \cap T$,

$$
\operatorname{sym}(x, S \cap T) \geq \min \{\operatorname{sym}(x, S), \operatorname{sym}(x, T)\}
$$

2. (Superminimality under Minkowski sums)

$$
\operatorname{sym}(x+y, S+T) \geq \min \{\operatorname{sym}(x, S), \operatorname{sym}(y, T)\}
$$


3. (Invariance under polarity)

$$
\operatorname{sym}(0, S-x)=\operatorname{sym}\left(0,(S-x)^{\circ}\right)
$$

4. (Minimality under Cartesian product)

$$
\operatorname{sym}((x, y), S \times T)=\min \{\operatorname{sym}(x, S), \operatorname{sym}(y, T)\}
$$

5. (Lower bound under affine transformation) Let $A(\cdot)$ be an affine transformation. Then

$$
\operatorname{sym}(A(x), A(S)) \geq \operatorname{sym}(x, S)
$$

with equality if $A(\cdot)$ is invertible.

Proof To prove 5, without loss of generality, we can translate the sets and suppose that $x=0$. Let $\alpha=\min \{\operatorname{sym}(0, S), \operatorname{sym}(0, T)\}$. Then $-\alpha S \subset S,-\alpha T \subset T$ which implies

$$
-\alpha(S \cap T)=-\alpha S \cap-\alpha T \subset S \cap T,
$$

and (5) is proved.

To prove (6), again, without loss of generality, we can translate both sets and suppose that $x=y=0$, and define $\alpha=\operatorname{sym}(0, S)$ and $\beta=\mathbf{s y m}(0, T)$. By definition, $-\alpha S \subset S$ and $-\beta T \subset T$. Then it follows trivially that

$$
-\alpha S-\beta T \subset(S+T) .
$$

Replacing $\alpha$ and $\beta$ by the minimum between them, the result follows.

In order to prove (7), we can assume $x=0$, then

$$
\operatorname{sym}(0, S)=\alpha \Rightarrow-\alpha S \subseteq S .
$$

Assuming $\operatorname{sym}\left(0, S^{\circ}\right)<\alpha$, there exist $\bar{y} \in S^{\circ}$ such that $-\alpha \bar{y} \notin S^{\circ}$.

Thus, there exists $x \in S,-\alpha \bar{y}^{T} x>1$. However, since $-\alpha x \in-\alpha S \subseteq S$, then

$$
-\alpha \bar{y}^{T} x=\bar{y}^{T}(-\alpha x) \leq 1, \quad \text { since } \quad \bar{y} \in S^{\circ},
$$

which is a contradiction. Thus

$$
\operatorname{sym}(0, S) \leq \mathbf{s y m}\left(0, S^{\circ}\right) \leq \mathbf{s y m}\left(0, S^{\circ \circ}\right)=\mathbf{s y m}(0, S) .
$$

Equality (8) is left as a simple exercise.

To prove inequality (9), we can assume that $A(\cdot)$ is a linear operator and that $x=0$ (since $\operatorname{sym}(x, S)$ is invariant under translation), and suppose that $\alpha<\operatorname{sym}(x, S)$. Then, $-\alpha S \subseteq S$ which implies that $A(-\alpha S) \subseteq A(S)$. Since $A(\cdot)$ is a linear operator, $A(-\alpha S)=-\alpha A(S) \subseteq A(S)$. It is straightforward to show that equality holds in (9) when $A(\cdot)$ is invertible. 
Remark 1 Unlike the case of affine transformation, $\operatorname{sym}(x, S)$ is not invariant under projective transformation. For instance, let $S=[-1,1] \times[-1,1]$ be unit cube, for which $\operatorname{sym}(S)=1$, and consider the projective transformation that maps $x \in \mathbb{R}^{2}$ to $x /\left(1+x_{2} / 3\right) \in \mathbb{R}^{2}$. Then, the symmetric set $S$ will be mapped to the trapezoid

$$
T:=\operatorname{conv}\left\{\left(\frac{3}{4}, \frac{3}{4}\right),\left(-\frac{3}{4}, \frac{3}{4}\right),\left(-\frac{3}{2},-\frac{3}{2}\right),\left(\frac{3}{2},-\frac{3}{2}\right)\right\},
$$

for which $\operatorname{sym}(T)<1$. This lack of invariance is used in [4] in the development of a methodology designed to improve the symmetry of a point in a set using a projective transformation.

\section{Geometric properties}

Whereas there always exists an $n$-rounding of a convex body $S \subset \mathbb{R}^{n}$, a symmetric convex body $S$ possesses some even more powerful geometric properties, for example there exists a $\sqrt{n}$-rounding of $S$ when $S$ is symmetric, see [15]. The geometric flavor of the definition of the symmetry function in (4) suggests that $\operatorname{sym}(\cdot, S)$ is connected to extensions of these geometric properties and gives rise to new properties as well; these properties are explored and developed in this section. We examine volumes of intersections of $S$ with halfspaces and halfplanes that cut through $x \in S$ in Sect. 3.1, notions of distance and symmetry in Sect. 3.2, set approximation results in Sect. 3.3, and results on probability and symmetry in Sect. 3.4.

\subsection{Volumes and symmetry}

We start with two theorems that connect $\operatorname{sym}(x, S)$ to bounds on the $n$-dimensional volume of the intersection of $S$ with a halfspace cut through $x$, and with the $(n-1)$-dimensional volume of the intersection of $S$ with a hyperplane passing through $S$. Similar results have been extensively used in the literature. For example, if $S$ is symmetric around some point $x^{*}$, it is clear that the intersection of $S$ with a halfspace cut through $x^{*}$ contains exactly one half of the volume of $S$. Moreover, it is well known that a halfspace cut through the center of mass generates a set with at least $1 / \mathbf{e}$ of the original volume, and this fact has been utilized in [5] to develop theoretically efficient probabilistic methods for solving convex optimization problems.

Let $v \in \mathbb{R}^{n}, v \neq 0$ be given, and for all $x \in S$ define $H(x):=\left\{z \in S: v^{T} z=\right.$ $\left.v^{T} x\right\}$ and $H^{+}(x):=\left\{z \in S: v^{T} z \leq v^{T} x\right\}$. Also let $\operatorname{Vol}_{n}(\cdot)$ denotes the volume measure on $\mathbb{R}^{n}$. We have:

Theorem 2 Under Assumption A, if $x \in S$, then

$$
\frac{\operatorname{sym}(x, S)^{n}}{1+\operatorname{sym}(x, S)^{n}} \leq \frac{\operatorname{Vol}_{n}\left(H^{+}(x)\right)}{\operatorname{Vol}_{n}(S)} \leq \frac{1}{1+\operatorname{sym}(x, S)^{n}} .
$$


Proof Without loss of generality, assume that $x$ is the origin and $\alpha=\operatorname{sym}(x, S)$. Define $K_{1}=H^{+}(x)$ and $K_{2}=S \backslash K_{1}$. Clearly, $\operatorname{Vol}_{n}\left(K_{1}\right)+\operatorname{Vol}_{n}\left(K_{2}\right)=\operatorname{Vol}_{n}(S)$. Notice that $-\alpha K_{2} \subset K_{1}$ and $-\alpha K_{1} \subset K_{2}$. Therefore

$$
\operatorname{Vol}_{n}(S) \geq \operatorname{Vol}_{n}\left(K_{1}\right)+\operatorname{Vol}_{n}\left(-\alpha K_{1}\right)=\operatorname{Vol}_{n}\left(K_{1}\right)\left(1+\alpha^{n}\right)
$$

which proves the second inequality. The first inequality follows easily from

$$
\operatorname{Vol}_{n}(S)=\operatorname{Vol}_{n}\left(K_{1}\right)+\operatorname{Vol}_{n}\left(K_{2}\right) \leq \operatorname{Vol}_{n}\left(K_{1}\right)+\frac{\operatorname{Vol}_{n}\left(K_{1}\right)}{\alpha^{n}}
$$

For the next theorem, define the function $f(x)=\operatorname{Vol}_{n-1}(H(x))^{1 /(n-1)}$ for all $x \in S$.

Theorem 3 Under Assumption A, for every point $x \in S$,

$$
\frac{f(x)}{\max _{y \in S} f(y)} \geq \frac{2 \operatorname{sym}(x, S)}{1+\operatorname{sym}(x, S)} .
$$

Proof Let $\alpha=\operatorname{sym}(x, S)$ and let $y^{*}$ satisfy $y^{*} \in \arg \max _{y} f(y)$. Note that

$$
x+\alpha\left(x-H\left(y^{*}\right)\right) \subset S,
$$

and the set on the left in this inclusion passes through $x+\alpha\left(x-y^{*}\right)$, and so $x+\alpha\left(x-H\left(y^{*}\right)\right) \subset H\left(x+\alpha\left(x-y^{*}\right)\right)$. Next, recall that the $(n-1)$-dimensional volume of a set $S$ is invariant under translations and $\operatorname{Vol}_{n-1}(a S)=a^{n-1} \operatorname{Vol}_{n-1}(S)$ for any set $S$ and positive scalar $a$. Therefore

$$
\begin{aligned}
\alpha f\left(y^{*}\right) & =\left(\operatorname{Vol}_{n-1}\left(x+\alpha\left(x-H\left(y^{*}\right)\right)\right)\right)^{1 /(n-1)} \leq\left(\operatorname{Vol}_{n-1}\left(H\left(x+\alpha\left(x-y^{*}\right)\right)\right)\right)^{1 /(n-1)} \\
& =f\left(x+\alpha\left(x-y^{*}\right)\right)
\end{aligned}
$$

Note that we can write

$$
x=\frac{\alpha}{1+\alpha} y^{*}+\frac{1}{1+\alpha}\left(x+\alpha\left(x-y^{*}\right)\right) .
$$

where $x+\alpha\left(x-y^{*}\right) \in S$.

Noting that $f(\cdot)$ is concave (this follows from the Brunn-Minkowski inequality [10]), we have:

$$
\begin{aligned}
f(x) & \geq \frac{\alpha}{1+\alpha} f\left(y^{*}\right)+\frac{1}{1+\alpha} f\left(x+\alpha\left(x-y^{*}\right)\right) \geq \frac{\alpha}{1+\alpha} f\left(y^{*}\right)+\frac{\alpha}{1+\alpha} f\left(y^{*}\right) \\
& =\frac{2 \alpha}{1+\alpha} f\left(y^{*}\right)
\end{aligned}
$$

where the second inequality follows from (12). 
Remark 2 We conjecture that any symmetry point $x^{*}$ satisfies

$$
\frac{f\left(x^{*}\right)}{\max _{y \in S} f(y)} \geq \frac{2}{3} .
$$

\subsection{Distance and symmetry}

If $S$ is a symmetric convex body, then it is a straightforward exercise to show that the symmetry point of $S$ is unique. Furthermore, if $S$ is nearly symmetric, intuition suggests that two points in $S$ with high symmetry values cannot be too far apart. The two theorems in this subsection quantify this intuition. Given $x, y \in S$ with $x \neq y$, let $p(x, y), q(x, y)$ be the pair of endpoints of the chord in $S$ passing through $x$ and $y$, namely:

$$
\begin{array}{ll}
p(x, y)=x+s(x-y) \in \partial S & \text { where } s \text { is a maximal scalar } \\
q(x, y)=y+t(y-x) \in \partial S & \text { where } t \text { is a maximal scalar. }
\end{array}
$$

Theorem 4 Under Assumption A, let $\|\cdot\|$ be any norm on $\mathbb{R}^{n}$. For any $x, y \in S$ satisfying $x \neq y$, let $\alpha=\operatorname{sym}(x, S)$ and $\beta=\operatorname{sym}(y, S)$. Then:

$$
\|x-y\| \leq\left(\frac{1-\alpha \beta}{1+\alpha+\beta+\alpha \beta}\right)\|p(x, y)-q(x, y)\| .
$$

Proof For convenience let us denote the quantities $p(x, y), q(x, y)$ by $p, q$, and note that the chord from $p$ to $q$ contains, in order, the points $p, x, y$, and $q$. It follows from the symmetry values of $x, y$ that

$$
\begin{aligned}
& \|p-x\| \geq \alpha\|q-x\|=\alpha(\|y-x\|+\|q-y\|) \quad \text { and } \\
& \|q-y\| \geq \beta\|p-y\|=\beta(\|y-x\|+\|p-x\|) .
\end{aligned}
$$

Multiplying the first inequality by $1+\beta$, the second inequality by $1+\alpha$, adding the result and rearranging yields:

$$
\begin{aligned}
(1+\alpha+\beta+\alpha \beta)\|x-y\| & \leq(1-\alpha \beta)(\|p-x\|+\|x-y\|+\|q-y\|) \\
& =(1-\alpha \beta)\|p-q\|
\end{aligned}
$$

which yields the desired result.

Another relative measure of distance is the "cross-ratio distance" with respect to $S$. Let $x, y \in S, x \neq y$, be given and let $s, t$ be as defined in (13); the cross-ratio distance is given by:

$$
d_{S}(x, y):=\frac{(1+t+s)}{t s}
$$


Theorem 5 Under Assumption A, for any $x, y \in S, x \neq y$, let $s, t$ be as defined in (13). Then

$$
d_{S}(x, y) \leq \frac{1}{\operatorname{sym}(x, S) \cdot \mathbf{s y m}(y, S)}-1
$$

Proof Let $\alpha=\operatorname{sym}(x, S)$ and $\beta=\operatorname{sym}(y, S)$. By definition of symmetry, $t \geq \beta(1+s)$ and $s \geq \alpha(1+t)$. Then

$$
\begin{aligned}
d_{S}(x, y) & =\frac{(1+t+s)}{t s} \leq \frac{(1+t+s)}{\alpha(1+s) \beta(1+t)} \\
& =\frac{1}{\alpha \beta} \frac{(1+t+s)}{(1+s+t+s t)}=\left(\frac{1}{\alpha \beta}\right) \frac{1}{1+\frac{1}{d_{S}(x, y)}} .
\end{aligned}
$$

Thus $d_{S}(x, y) \leq \frac{1}{\alpha \beta}-1$.

We end this subsection with a comment on a question posed by Hammer in [14]: what is the upper bound on the difference between $\mathbf{s y m}(S)$ and $\operatorname{sym}\left(x^{c}, S\right)$, where $x^{c}$ is the centroid (center of mass) of $S$ ? It is well known that $\operatorname{sym}\left(x^{c}, S\right) \geq 1 / n$, see [14], and it follows trivially from the Löwner-John theorem that $\operatorname{sym}(S) \geq 1 / n$ as well. Now let $S$ be the Euclidean half-ball: $S:=\left\{x \in \mathbb{R}^{n}:\langle x, x\rangle \leq 1, x_{1} \geq 0\right\}$. It is an easy exercise to show that the unique symmetry point of $S$ is $x^{*}=(\sqrt{2}-1) e^{1}$ and that $\operatorname{sym}(S)=\frac{1}{\sqrt{2}}$, and so in this case $\operatorname{sym}(S)$ is a constant independent of the dimension $n$. On the other hand, $\operatorname{sym}\left(x^{c}, S\right)=\Omega\left(\frac{1}{\sqrt{n}}\right)$ (see [2]), and so for this class of instances the symmetry of the centroid is substantially less than the symmetry of the set for large $n$. For an arbitrary convex body $S$, note that in the extreme cases where $\operatorname{sym}(S)=1$ or $\operatorname{sym}(S)=1 / n$ the difference between $\operatorname{sym}(S)$ and $\operatorname{sym}\left(x^{c}, S\right)$ is zero; we conjecture that tight bounds on this difference are only small when $\operatorname{sym}(S)$ is either very close to 1 or very close to $1 / n$.

\subsection{Set-approximation and symmetry}

In this subsection we examine the approximation of the convex set $S$ by another convex set $P$. We say that $P$ is a $\beta$-approximation of $S$ if there exists a point $x \in S$ such that $\beta P \subset S-x \subset P$. In the case when $P$ is an ellipsoid centered at the origin, then the statement " $P$ is a $\beta$-approximation of $S$ " is equivalent to " $\beta P$ provides a $\frac{1}{\beta}$-rounding of $S$." We examine the interrelationship between the symmetry function and bounds on $\beta$-approximations for $S$ in the following three theorems.

A classical example of $\beta$-approximation is given by the Löwner-John theorem [15], which guarantees a $1 / \sqrt{n}$-approximation for a symmetric convex body and a $1 / n$-approximation for general convex body using ellipsoids. Unfortunately, the theorem does not provide more precise bounds for case when $S$ is nearly 
symmetric, i.e., $\operatorname{sym}(S)=1-\varepsilon$ for $\varepsilon$ small. This is partially rectified in the fourth result of this subsection, Theorem 9 .

Theorem 6 Under Assumption $A$, let $P$ be a convex body that is a $\beta$-approximation of $S$, and suppose that $\operatorname{sym}(0, P)=\alpha$. Then, $\operatorname{sym}(S) \geq \beta \alpha$.

Proof By definition we have $\beta P \subset S-x \subset P$ for some $x \in S$. Since $\operatorname{sym}(\cdot, \cdot)$ is invariant under translations, we can assume that $x=0$. Since $\operatorname{sym}(0, P)$ is invariant under nonzero scalings of $P$, we have

$$
-\alpha \beta S \subset-\alpha \beta P \subset \beta P \subset S
$$

Theorem 7 Under Assumption A, suppose that $x \in$ int $S$. Then there exists an ellipsoid E centered at 0 such that

$$
E \subset S-x \subset\left(\frac{\sqrt{n}}{\operatorname{sym}(x, S)}\right) E .
$$

Proof Suppose without loss of generality that $x=0$ (otherwise we translate $S$ ), and let $\alpha=\operatorname{sym}(0, S)$. Clearly, $-\alpha S \subset S$, and $\alpha S \subset S$. Consider a $\sqrt{n}$-rounding $E$ of $S \cap(-S)$. Then $\alpha S \subset S \cap(-S) \subset \sqrt{n} E \subset \sqrt{n} S$.

Theorem 8 Let $\|\cdot\|$ be any norm on $\mathbb{R}^{n}$, and let $B(x, r)$ denote the ball centered at $x$ with radius $r$. Under Assumption A, suppose that

$$
B(x, r) \subset S \subset P \subset S+B(0, \delta)
$$

for some $r$ and $\delta$ with $0<\delta<r$. Then

$$
\left(1-\frac{\delta}{r}\right) \leq \frac{\operatorname{sym}(x, S)}{\operatorname{sym}(x, P)} \leq\left(\frac{1}{1-\delta / r}\right)
$$

Proof Let $\alpha=\operatorname{sym}(x, P)$. Consider any chord of $P$ that passes through $x$, dividing the chord into two segments. Assume that the length of one segment is $\Delta$, then the length of the other segment must be at most $\Delta / \alpha$. It then follows that the length of the first segment of this chord in $S$ must be at least $\Delta-\delta$, while the length of the second segment of this chord in $S$ must be at most $\Delta / \alpha$. Since these inequalities hold for any chord, it follows that

$$
\operatorname{sym}(x, S) \geq \frac{\Delta-\delta}{\Delta / \alpha}=\alpha\left(1-\frac{\delta}{\Delta}\right) \geq \alpha\left(1-\frac{\delta}{r}\right)
$$

where the last inequality follows since $\Delta \geq r$, thereby showing that $\operatorname{sym}(x, S) \geq$ $\operatorname{sym}(x, P)\left(1-\frac{\delta}{r}\right)$. Note also that:

$$
B(x, r) \subset P \subset S+B(0, \delta) \subset P+B(0, \delta) .
$$


Letting $P$ play the role of $S$ in (16) and $S+B(0, \delta)$ play the role of $P$ in (16), it also follows from (17) that

$$
\operatorname{sym}(x, P) \geq \operatorname{sym}(x, S+B(0, \delta))\left(1-\frac{\delta}{r}\right) .
$$

However, using the superminimality of of $\operatorname{sym}(\cdot, \cdot)$ under Minkowski sums (6) of Theorem 3, we have

$$
\operatorname{sym}(x, S+B(0, \delta)) \geq \min \{\operatorname{sym}(x, S), \operatorname{sym}(0, B(0, \delta))\}=\operatorname{sym}(x, S),
$$

which when combined with the previous inequality completes the proof.

The center $x^{L}$ of the minimum-volume ellipsoid $E$ containing $S$ is called the Löwner-John center of $S$, and John showed in [15] that $E$ provides a $\sqrt{n}$-rounding of $S$ in the case when $S$ is symmetric and an $n$-rounding of $S$ when $S$ is not symmetric. The following theorem provides a sharpening of this result:

Theorem 9 Under Assumption A, let $E$ be the minimum volume ellipsoid containing $S$, and let $x^{L}$ be the Löwner-John center of S. Then E provides a $\sqrt{\frac{n}{\operatorname{sym}\left(x^{L}, S\right)}}$ rounding of $S$.

Remark 3 It follows from Theorem 9 that

$$
\operatorname{sym}\left(x^{L}, S\right) \geq \sqrt{\frac{\operatorname{sym}\left(x^{L}, S\right)}{n}}
$$

and hence $\operatorname{sym}\left(x^{L}, S\right) \geq 1 / n$. This in turn yields the Löwner-John result [13] that the rounding in the theorem is an $n$-rounding, and hence $\operatorname{sym}(S) \geq \mathbf{s y m}\left(x^{L}, S\right) \geq$ $1 / n$. Noting that when $S$ is symmetric the Löwner-John center must also be the symmetry point of $S$, it also follows from Theorem 9 that $S$ admits a $\sqrt{n}$-rounding when $\operatorname{sym}(S)=1$.

Remark 4 Theorem 7 is valid for every point in $S$ and Theorem 9 focuses on the Löwner-John center. We conjecture that Theorem 9 can be strengthened to prove the existence of a $\left(\sqrt{\frac{n}{\operatorname{sym}(S)}}\right)$-rounding of $S$.

The proof of Theorem 9 is based in part on ideas communicated to the second author by Earl Barnes [3] in 1998. We start with the following two elementary propositions:

Proposition 4 Let $w_{1}, \ldots, w_{k}$ be scalars and define $w_{\min }, w_{\max }$ to be the smallest and largest values among these scalars. For any $p \in \mathbb{R}^{k}$ satisfying $p \geq 0$ and $e^{T} p=$ 1 define $\mu=p^{T} w$ and $\sigma^{2}=\sum_{i=1}^{k} p_{i}\left(w_{i}-\mu\right)^{2}$. Then $\left(w_{\max }-\mu\right)\left(\mu-w_{\min }\right) \geq \sigma^{2}$. 
Proof Clearly, $\sum_{i=1}^{k} p_{i}\left(w_{\max }-w_{i}\right)\left(w_{i}-w_{\min }\right) \geq 0$. Therefore $\mu w_{\max }+\mu w_{\min }-$ $\sum_{i=1}^{k} p_{i} w_{i}^{2}-w_{\min } w_{\max } \geq 0$. It then follows that $\left(w_{\max }-\mu\right)\left(\mu-w_{\min }\right)=\mu w_{\max }+$ $\mu w_{\min }-\mu^{2}-w_{\min } w_{\max } \geq \sum_{i=1}^{k} p_{i} w_{i}^{2}-\mu^{2}=\sigma^{2}$.

Proposition 5 Let $y^{1}, \ldots, y^{k} \in \mathbb{R}^{n}$ be given, let $p \in \mathbb{R}^{k}$ satisfy $p \geq 0$ and $e^{T} p=1$, and suppose that $\sum_{i=1}^{n} p_{i} y^{i}=0$ and $\sum_{i=1}^{k} p_{i} y^{i}\left(y^{i}\right)^{T}=\frac{1}{n} I$. Then for any $\bar{b} \in \mathbb{R}^{n}$ with $\|\bar{b}\|_{2}=1$ it holds that

$$
\max _{i=1, \ldots, k} \bar{b}^{T} y^{i} \geq \sqrt{\frac{\operatorname{sym}\left(0, \operatorname{conv}\left(\left\{y^{i}\right\}_{i=1}^{k}\right)\right)}{n}} .
$$

Proof Let $\bar{b} \in \mathbb{R}^{n}$ satisfying $\|\bar{b}\|_{2}=1$ be given, and define $w_{i}=\bar{b}^{T} y^{i}$. Then

$$
\mu=\sum_{i=1}^{k} p_{i} w_{i}=\sum_{i=1}^{k} p_{i} \bar{b}^{T} y^{i}=\bar{b}^{T}\left(\sum_{i=1}^{k} p_{i} y^{i}\right)=0
$$

and

$$
\sigma^{2}=\sum_{i=1}^{k} p_{i}\left(w_{i}-\mu\right)^{2}=\sum_{i=1}^{k} p_{i} w_{i}^{2}=\sum_{i=1}^{k} p_{i} \bar{b}^{T} y^{i}\left(y^{i}\right)^{T} \bar{b}=\frac{1}{n} \bar{b}^{T} I \bar{b}=\frac{1}{n}
$$

It then follows from Proposition 4 that $\left(\max _{i} w_{i}\right)\left(-\min _{i} w_{i}\right)=\left(\max _{i} w_{i}-\mu\right)(\mu-$ $\left.\min _{i} w_{i}\right) \geq \sigma^{2}=\frac{1}{n}$. Let $\alpha:=\operatorname{sym}\left(0, \operatorname{conv}\left(\left\{y^{i}\right\}_{i=1}^{k}\right)\right)$, and notice that $-\min _{i} \bar{b}^{T} y^{i} \leq$ $\frac{1}{\alpha} \max _{i} \bar{b}^{T} y^{i}$. Therefore

$$
\frac{1}{n} \leq \max _{i} w_{i}\left(-\min _{i} w_{i}\right) \leq \max _{i} w_{i}\left(\frac{1}{\alpha} \max _{i} w_{i}\right)=\frac{\left(\max _{i} w_{i}\right)^{2}}{\alpha}
$$

from which the result readily follows.

Proof of Theorem 9 We first suppose that $S$ is the convex hull of finitely many points, and we write $S=\operatorname{conv}\left(\left\{v^{i}\right\}_{i=1}^{k}\right)$. The minimum volume ellipsoid containing $S$ is obtained using the solution of the following optimization problem:

$$
\begin{array}{ll}
\min _{Q, c}-\ln \operatorname{det} Q \\
\text { s.t. } \quad\left(v^{i}-c\right)^{T} Q\left(v^{i}-c\right) \leq 1, \quad i=1, \ldots, k \\
& Q \succeq 0 .
\end{array}
$$

If $Q, c$ solves (18) then $E^{O}:=\left\{x \in \mathbb{R}^{n}:(x-c)^{T} Q(x-c) \leq 1\right\}$ is the minimum volume ellipsoid containing $S$ and $c$ is the Löwner-John center. Letting $E^{I}:=\left\{x \in \mathbb{R}^{n}:(x-c)^{T} Q(x-c) \leq \frac{\alpha}{n}\right\}$ where $\alpha:=\operatorname{sym}(c, S)$, we need to show that $E^{I} \subset S$. Equivalently, for every $b \in \mathbb{R}^{n}$ we need to show that

$$
\max \left\{b^{T} x: x \in E^{I}\right\} \leq \max \left\{b^{T} x: x \in S\right\} .
$$


The KKT conditions for (18) are necessary and sufficient, see John [15], and can be written as:

$$
\begin{aligned}
& -Q^{-1}+\sum_{i=1}^{k} \lambda_{i}\left(v^{i}-c\right)\left(v^{i}-c\right)^{T}=0 \\
& \sum_{i=1}^{k} \lambda_{i} Q\left(v^{i}-c\right)=0 \\
& \lambda_{i} \geq 0, \quad i=1, \ldots, k \\
& \left(v^{i}-c\right)^{T} Q\left(v^{i}-c\right) \leq 1, \quad i=1, \ldots, k \\
& \lambda_{i}\left(v^{i}-c\right)^{T} Q\left(v^{i}-c\right)=\lambda_{i}, \quad i=1, \ldots, k \\
& Q \succ 0 .
\end{aligned}
$$

Defining $y^{i}=Q^{1 / 2}\left(v^{i}-c\right)$ and $p_{i}=\frac{\lambda_{i}}{n}$ we have $p \geq 0$, and using the KKT conditions we obtain:

$$
\begin{aligned}
n=\operatorname{trace}(I) & =\operatorname{trace}\left(Q^{1 / 2} Q^{-1} Q^{1 / 2}\right) \\
& =\sum_{i=1}^{k} \lambda_{i} \operatorname{trace}\left(Q^{1 / 2}\left(v^{i}-c\right)\left(v^{i}-c\right)^{T} Q^{1 / 2}\right) \\
& =\sum_{i=1}^{k} \lambda_{i} \operatorname{trace}\left(\left(v^{i}-c\right)^{T} Q^{1 / 2} Q^{1 / 2}\left(v^{i}-c\right)\right) \\
& =\sum_{i=1}^{k} \lambda_{i}\left(v^{i}-c\right)^{T} Q^{1 / 2} Q^{1 / 2}\left(v^{i}-c\right) \\
& =\sum_{i=1}^{k} \lambda_{i}=e^{T} \lambda,
\end{aligned}
$$

and it follows that $e^{T} p=\frac{e^{T} \lambda}{n}=1$. Furthermore,

$$
\sum_{i=1}^{k} p_{i} y^{i}=\frac{1}{n} Q^{-1 / 2} \sum_{i=1}^{k} \lambda_{i} Q\left(v^{i}-c\right)=0
$$

and

$$
\sum_{i=1}^{k} p_{i} y^{i}\left(y^{i}\right)^{T}=\sum_{i=1}^{k} \frac{\lambda_{i}}{n} Q^{1 / 2}\left(v_{i}-c\right)\left(v_{i}-c\right)^{T} Q^{1 / 2}=\frac{1}{n} I .
$$


For any $b \in \mathbb{R}^{n}, b \neq 0$, define $\bar{b}:=\frac{Q^{-1 / 2} b}{\sqrt{b^{T} Q^{-1} b}}$ and note that $\|\bar{b}\|_{2}=1$. Then $p, y^{1}, \ldots, y^{k}$, and $\bar{b}$ satisfy the hypotheses of of Proposition 5 , and so

$$
\begin{aligned}
\max \left\{b^{T} x: x \in S\right\} & =\max _{i} b^{T} v^{i} \\
& =b^{T} c+\sqrt{b^{T} Q^{-1} b}\left(\max _{i} \bar{b}^{T} Q^{1 / 2}\left(v^{i}-c\right)\right) \\
& =b^{T} c+\sqrt{b^{T} Q^{-1} b}\left(\max _{i} \bar{b}^{T} y^{i}\right) \\
& \geq b^{T} c+\sqrt{\frac{\alpha}{n}} \sqrt{b^{T} Q^{-1} b}
\end{aligned}
$$

where the inequality is from Proposition 5, and we use the fact that $\operatorname{sym}\left(0, \operatorname{conv}\left(\left\{v^{i}\right\}_{i=1}^{k}\right)\right)=\operatorname{sym}\left(0, \operatorname{conv}\left(\left\{y^{i}\right\}_{i=1}^{k}\right)\right)$ which follows from the invariance of $\operatorname{sym}(\cdot, \cdot)$ under invertible affine transformation, see (5.) of Theorem 2. On the other hand we have:

$\max \left\{b^{T} x: x \in E^{I}\right\}=\max \left\{b^{T} x:(x-c)^{T} Q(x-c) \leq \frac{\alpha}{n}\right\}=b^{T} c+\sqrt{\frac{\alpha}{n}} \sqrt{b^{T} Q^{-1} b}$,

which then yields $\max \left\{b^{T} x: x \in E^{I}\right\} \leq \max \left\{b^{T} x: x \in S\right\}$, proving the result under the hypothesis that $S$ is the convex hull of finitely many points.

Finally, suppose $S$ is not the convex hull of finitely many points. For any $\delta>0$ there is a polytope $P_{\delta}$ that approximates $S$ in the sense that $S \subset P_{\delta} \subset S+B(0, \delta)$, where $B(0, \delta)$ is the ball of radius $\delta$ centered at 0 . Limiting arguments can then be used to show the result by taking a limiting sequence of polytopes $P_{\delta}$ as $\delta \rightarrow 0$ and noticing from Theorem 8 that $\lim _{\delta \rightarrow 0} \operatorname{sym}\left(0, P_{\delta}\right)=\operatorname{sym}(0, S)$.

We close this subsection by discussing a norm closely related to the symmetry function that was also used in [9]. Without loss of generality, assume that $x^{*}=0$ is a symmetry point of $S$ and define the following norm associated with $S$ :

$$
\|x\|_{S}=\min _{t}\{t: x \in t(S \cap-S)\}
$$

and let $B_{S}(c, r)$ denote the ball of radius $r$ centered at $c$ using the norm defined in (19).

Lemma 1 Under Assumption A, suppose that $x^{*}=0$ is a symmetry point of $S$. Then

$$
B_{S}(0,1) \subset S \subset B_{S}(0,1 / \mathbf{s y m}(S))
$$

Proof By construction, $B_{S}(0,1)=S \cap-S \subset S$. For the second inclusion, observe that $-\operatorname{sym}(S) S \subset S$, which then implies that $S \subset-\frac{1}{\operatorname{sym}(S)} S$. Therefore $S \subset \frac{1}{\operatorname{sym}(S)}(S \cap-S)$.

Remark 5 The norm defined by (19) induces the best $\beta$-approximation among all norms in $\mathbb{R}^{n}$. That is, $\left(0,1,1 / \mathbf{s y m}(S),\|\cdot\|_{S}\right)$ solves the following optimization 
problem

$$
\min _{x, r, R,\|\cdot\|}\left\{\frac{R}{r}: B_{\|\cdot\|}(x, r) \subset S \subset B_{\|\cdot\|}(x, R)\right\}
$$

Proof Suppose that there exists a norm $\|\cdot\|, r, R$, and $x$, such that

$$
B_{\|\cdot\|}(x, r) \subset S \subset B_{\|\cdot\|}(x, R)
$$

and $\frac{R}{r}<\frac{1}{\operatorname{sym}(S)}$. Using Theorem 6, we have $\operatorname{sym}(x, S) \geq \frac{r}{R}>\operatorname{sym}(S)$, a contradiction.

\subsection{Probability and symmetry}

This subsection contains two results related to symmetry and probability. To set the stage for the first result, suppose that $X$ is a random vector uniformly distributed on the given convex body $S \subset \mathbb{R}^{n}$. Theorem 10 gives lower bounds on the expected value of $\operatorname{sym}(X, S)$ and on the probability that $\operatorname{sym}(X, S)$ will be larger than a constant fraction $1 / M$ of $\mathbf{s y m}(S)$. Roughly speaking, Theorem 10 states that it is likely that $\operatorname{sym}(X, S)$ is relatively large. The second result, Theorem 11, is an extension of Anderson's Lemma [1] concerning the integral of a nonnegative logconcave even function on $S$, and has many statistical applications.

Theorem 10 Under Assumption A, let $X$ be a random vector uniformly distributed on S. Then

(i) $\mathbb{E}[\mathbf{s y m}(X, S)] \geq \frac{\operatorname{sym}(S)}{2 n+1}$

(ii) For any $M \geq 1, \operatorname{Pr}\left(\operatorname{sym}(X, S) \geq \frac{\operatorname{sym}(S)}{M}\right) \geq\left(1-\frac{2}{M+1}\right)^{n}$

(iii) Among symmetric sets $S, \mathbb{E}[\operatorname{sym}(X, S)]$ and $\operatorname{Pr}\left(\operatorname{sym}(X, S) \geq \frac{1}{M}\right)$ are functions only of the dimension $n$ and are independent of the specific set $S$, and satisfy:

$$
\begin{aligned}
& \text { (iii.a) } \mathbb{E}[\operatorname{sym}(X, S)] \leq \frac{1}{2(n+1)}+\frac{1}{(n+1)(n+2)} \\
& \text { (iii.b) For any } M \geq 1, \operatorname{Pr}\left(\operatorname{sym}(X, S) \geq \frac{1}{M}\right)=\left(1-\frac{2}{M+1}\right)^{n} .
\end{aligned}
$$

Proof Without loss of generality we assume for convenience that $x^{*}=0$ is a symmetry point of $S$. Let $t \in[0,1]$. For any $x \in t S$, consider any chord of $S$ that intersects $x$, and let $p, q$ be the endpoints of this chord. Note that $\|p\|_{S} \leq 1 / \operatorname{sym}(S)$ and $\|x\|_{S} \leq t / \mathbf{s y m}(S)$, where $\|\cdot\|_{S}$ is the norm defined in (19). Also, it follows from basic convexity that $t S+(1-t) B_{S}(0,1) \subset t S+(1-t) S \subset S$, 
where $B_{S}(0,1)$ is the unit ball centered at the origin for the norm $\|\cdot\|_{S}$. This then implies that if $x \in t S$ and $q \in \partial S$ then $\|q-x\|_{S} \geq 1-t$. Therefore

$$
\frac{\|q-x\|_{S}}{\|p-x\|_{S}} \geq \frac{1-t}{\|p\|_{S}+\|x\|_{S}} \geq \frac{1-t}{1 / \operatorname{sym}(S)+t / \operatorname{sym}(S)},
$$

which implies that

$$
\operatorname{sym}(x, S) \geq \operatorname{sym}(S) \frac{1-t}{1+t} .
$$

Now suppose that $X$ is a random vector uniformly distributed on $S$, and consider the random variable $t(X)$ defined uniquely by the inclusion $X \in \partial(t S)$. Then

$$
P(t(X) \leq t)=P(X \in t S)=\frac{\operatorname{Vol}(t S)}{\operatorname{Vol}(S)}=t^{n}
$$

which implies that the density of $t(X)$ is given simply by $f(t)=n t^{n-1}$. Therefore using (20) we have:

$$
\begin{aligned}
\mathbb{E}[\mathbf{s y m}(X, S)] & \geq \mathbb{E}\left[\operatorname{sym}(S) \frac{1-t(X)}{1+t(X)}\right] \\
& =\int_{0}^{1} \mathbf{s y m}(S) \frac{1-t}{1+t} n t^{n-1} \mathrm{~d} t \\
& =n \mathbf{s y m}(S) \int_{0}^{1} \frac{1-t}{1+t} t^{n-1} \mathrm{~d} t \\
& \geq n \mathbf{s y m}(S) \int_{0}^{1} t^{n-1}(1-\sqrt{t}) \mathrm{d} t \\
& =\frac{\operatorname{sym}(S)}{2 n+1},
\end{aligned}
$$

where the second inequality follows from the observation that $\frac{1-t}{1+t} \geq 1-\sqrt{t}$ for $t \in[0,1]$. This proves $(i)$.

To prove (ii), let $M \geq 1$ be given and define $\bar{t}:=1-\frac{2}{M+1}$ and note the relationship

$$
\frac{1-\bar{t}}{1+\bar{t}}=\frac{1}{M}
$$

Since $\{x \in S: x \in \bar{t} S\} \subset\left\{x \in S: \operatorname{sym}(x, S) \geq \frac{\operatorname{sym}(S)}{M}\right\}$ from (20), we have:

$$
\operatorname{Pr}\left(\operatorname{sym}(X, S) \geq \frac{\operatorname{sym}(S)}{M}\right) \geq \operatorname{Pr}(X \in \bar{t} S)=(\bar{t})^{n},
$$


which establishes (ii). To prove (iii) notice from Proposition 2 that (20) holds with equality in this case, whereby the above derivations yield $\mathbb{E}[\operatorname{sym}(X, S)]=$ $n \int_{0}^{1} \frac{1-t}{1+t} t^{n-1} \mathrm{~d} t$ and $\operatorname{Pr}\left(\operatorname{sym}(X, S) \geq \frac{1}{M}\right)=(\bar{t})^{n}$, which are functions of $n$ and are independent of $S$, thus showing (iii) and (iii.b). Noting that $\frac{1-t}{1+t} \leq 1-\frac{3}{2} t+\frac{1}{2} t^{2}$ for $t \in[0,1]$, we obtain in the symmetric case that

$$
\mathbb{E}[\mathbf{s y m}(X, S)] \leq n \int_{0}^{1}\left(1-\frac{3}{2} t+\frac{1}{2} t^{2}\right) t^{n-1} \mathrm{~d} t=\frac{1}{2(n+1)}+\frac{1}{(n+1)(n+2)},
$$

which shows (iii.a).

Corollary 1 Let $X$ be a random vector uniformly distributed on $S \subset \mathbb{R}^{n}$ for $n \geq 2$. Then

$$
\operatorname{Pr}\left(\operatorname{sym}(X, S) \geq \frac{\operatorname{sym}(S)}{n}\right) \geq\left(1-\frac{2}{n+1}\right)^{n} \geq 1 / 9,
$$

and the lower bound goes to $1 /(\mathbf{e})^{2}$ as $n \rightarrow \infty$.

The following is an extension of Anderson's Lemma [1], whose proof relies on the Brunn-Minkowski inequality in the symmetric case.

Theorem 11 Let $S \subset \mathbb{R}^{n}$ be a compact convex set which contains the origin in its interior, and let $\alpha=\mathbf{s y m}(0, S)$. Let $f(\cdot)$ be a nonnegative quasiconcave even function that is Lebesgue integrable. Then for $0 \leq \beta \leq 1$ and any $y \in \mathbb{R}^{n}$,

$$
\int_{S} f(x+\beta y) \mathrm{d} x \geq \alpha^{n} \int_{S} f\left(x+\frac{y}{\alpha}\right) \mathrm{d} x .
$$

Proof We refer to [7] for a proof in the symmetric case $\alpha=1$. Suppose that $f(\cdot)$ is an indicator function of a set $K$. This implies that $K$ is convex and $\operatorname{sym}(0, K)=1$. Therefore:

$$
\begin{aligned}
\int_{S} f(x+\beta y) \mathrm{d} x & \geq \int_{S \cap-S} f(x+\beta y) \mathrm{d} x \\
& \geq \int_{S \cap-S} f(x+y) \mathrm{d} x \\
& =\operatorname{Vol}_{n}((S \cap-S) \cap(K-y))=\operatorname{Vol}_{n}\left((S \cap-S) \cap \alpha\left(\frac{K-y}{\alpha}\right)\right) \\
& \geq \operatorname{Vol}_{n}\left(\alpha S \cap \alpha\left(K-\frac{y}{\alpha}\right)\right)=\alpha^{n} \operatorname{Vol}_{n}\left(S \cap\left(K-\frac{y}{\alpha}\right)\right)
\end{aligned}
$$

where the second inequality follows from Anderson's original theorem [1], and the third inequality holds simply because $\alpha S \subset S \cap-S$ and $K \subset \frac{K}{\alpha}$. Thus the 
result is true for simple quasiconcave even functions, and using standard arguments of dominated and monotone convergence, the result also holds for all nonnegative quasiconcave even Lebesgue-integrable functions.

The following corollary shows the potential usefulness of Theorem 11 in probability theory. We note that the density function of a uniformly distributed or an $n$-dimensional Gaussian random vector with mean $\mu=0$ satisfies the functional conditions of Theorem 11.

Corollary 2 Let $X$ be a random variable in $\mathbb{R}^{n}$ whose density function $f(\cdot)$ is an even quasiconcave function. In addition, let $Y$ be an arbitrary random vector independent of $X$, and let $\beta \in[0,1]$. If $S \subset \mathbb{R}^{n}$ is a compact convex set which contains the origin in its interior and $\alpha=\operatorname{sym}(0, S)$, then

$$
\operatorname{Pr}(X+\beta Y \in S) \geq \alpha^{n} \operatorname{Pr}\left(X+\frac{Y}{\alpha} \in S\right) .
$$

Proof Noting that $\alpha$ does not depend on $Y$, we have:

$$
\begin{aligned}
\operatorname{Pr}(X+\beta Y \in S) & =\iint_{S-\beta y} f(x) \mathrm{d} x \mathrm{~d} \operatorname{Pr}(y)=\iint_{S} f(x-\beta y) \mathrm{d} x \mathrm{~d} \operatorname{Pr}(y) \\
& \geq \alpha^{n} \iint_{S} f\left(x-\frac{y}{\alpha}\right) \mathrm{d} x \mathrm{~d} \operatorname{Pr}(y)=\alpha^{n} \operatorname{Pr}\left(X+\frac{Y}{\alpha} \in S\right) .
\end{aligned}
$$

\section{Characterization of symmetry points via the normal cone}

Let $\mathrm{S}_{\text {opt }}(S)$ denote the set of symmetry points of the convex body $S$. In this section we provide a characterization of $\mathrm{S}_{\mathrm{opt}}(S)$. From (4) and (2) we see that $\mathrm{S}_{\mathrm{opt}}(S)$ is the $x$-part of the optimal solution of:

$$
\begin{aligned}
\operatorname{sym}(S)=\max _{x, \alpha} & \alpha \\
\text { s.t. } & \alpha(x-S) \subseteq(S-x) \\
& \alpha \geq 0 .
\end{aligned}
$$

For any given $x \in S$ let $\alpha=\operatorname{sym}(x, S)$. Motivated by the set-containment definition of $\operatorname{sym}(x, S)$ in (4), let $V(x)$ denote those points $v \in \partial S$ that are also elements of the set $x+\alpha(x-S)$. We call these points the "touching points" of $x$ in $S$, namely:

$$
V(x):=\partial S \cap(x+\alpha(x-S)) \quad \text { where } \alpha=\operatorname{sym}(x, S) .
$$

Let $N_{S}(y)$ denote the normal cone map for points $y \in S$. We assemble the union of all normal cone vectors of all of the touching points of $x$ and call the resulting set the "support vectors" of $x$ : 


$$
S V(x)=\left\{s \in \mathbb{R}^{n}:\|s\|_{2}=1 \text { and } s \in N_{S}(v) \text { for some } v \in V(x)\right\} .
$$

The following characterization theorem essentially states that $x^{*} \in S$ is a symmetry point of $S$ if and only if the origin is in the convex hull of the support vectors of $x$ :

Theorem 12 Under Assumption A, let $x^{*} \in S$. The following statements are equivalent:

(i) $x^{*} \in S_{\text {opt }}(S)$

(ii) $0 \in \operatorname{conv} S V\left(x^{*}\right)$.

The proof of Theorem 12 we will rely on the following technical result:

Lemma 2 Suppose that $S$ is a convex body in a Euclidean space and $x \in \operatorname{int} S$ and $\alpha \geq 0$. Then $\alpha<\operatorname{sym}(x, S)$ if and only if $\alpha(x-S) \subseteq \operatorname{int}(S-x)$.

Proof $(\Rightarrow)$ The case $\alpha=0$ is trivial. For $\alpha>0$, since $x \in \operatorname{int} S$ and $S$ is a convex body, $\alpha<\operatorname{sym}(x, S)$ implies that

$$
\alpha(x-S) \subset \operatorname{sym}(x, S) \operatorname{int}(x-S) \subseteq \operatorname{int}(S-x) .
$$

$(\Leftarrow)$ For a fixed value of $\alpha$, rearrange the subset system to be: $C:=x+\alpha(x-$ $S) \subset$ int $S$. However, $S$ is a compact set, whereby $\alpha$ can be increased to $\alpha+\varepsilon$ for some small positive value of $\epsilon$ and still maintain $x+(\alpha+\varepsilon)(x-S) \subset \operatorname{int} S \subset S$, which by (4) is equivalent to $\operatorname{sym}(x, S) \geq \alpha+\varepsilon$.

The proof of Theorem 12 will also use the following construction:

Lemma 3 Consider the function $f(\cdot): \mathbb{R}^{n} \rightarrow \mathbb{R}$ defined as

$$
f(x)=\sup _{\substack{y \in \partial S \\ s \in N_{S}(y) \\\|s\|_{2}=1}}\langle s, x-y\rangle .
$$

Then

(i) $f(\cdot)$ is convex,

(ii) $f(x)=0$ for $x \in \partial S$,

(iii) $f(x)>0$ for $x \notin S$,

(iv) $f(x)<0$ for $x \in \operatorname{int} S$, and

(v) $\left\{s:\|s\|_{2}=1, s \in N_{S}(x)\right\} \subset \partial f(x)$ for $x \in \partial S$.

Proof As the supremum of affine functions, $f(\cdot)$ is convex, which shows (i). For $x \in \partial S, f(x) \geq 0$. For $(y, s)$ feasible for (28), $\langle s, x-y\rangle \leq 0$ for all $x \in S$ by definition of the normal cone, whereby $f(x)=0$, which shows (ii). For $x \in \operatorname{int} S$, there exists $\delta>0$ such that $B_{2}(x, \delta) \subset S$. Let $(y, s)$ be feasible for (28), then $\langle s, x-y\rangle=\langle s,(x+\delta s-y)-\delta s\rangle \leq\langle s,-\delta s\rangle=-\delta$, which then implies that $f(x) \leq-\delta$ and shows (iv). 
For $x \notin S$, there exists a hyperplane strictly separating $x$ from $S$. That is, there exists $\bar{s}$ satisfying $\|\bar{s}\|_{2}=1$ such that $\langle\bar{s}, x\rangle>\max _{y}\{\langle\bar{s}, y\rangle: y \in S\}$, and let $\bar{y}$ be an optimal solution of this problem. Then $(\bar{y}, \bar{s})$ is feasible for (28) and it follows that $f(x) \geq\langle\bar{s}, x-\bar{y}\rangle>0$, showing (iii). For $x \in \partial S$ and any $s \in N_{S}(x)$ satisfying $\|s\|_{2}=1$, it follows that for all $w$ that $f(w) \geq\langle s, w-x\rangle=f(x)+\langle s, w-x\rangle$, thereby showing (v).

Proof of Theorem 12 Suppose that $x^{*} \in \mathrm{S}_{\mathrm{opt}}(S)$. From (4) and Lemma 3 it follows that $x^{*}$ is a solution together with $\alpha^{*}:=\operatorname{sym}(S)$ of the following optimization problem:

$$
\begin{aligned}
\operatorname{sym}(S)= & \max _{x, \alpha} \alpha \\
\text { s.t. } & f(x-\alpha(y-x)) \leq 0 \quad \text { for all } y \in S .
\end{aligned}
$$

The necessary optimality conditions for this problem imply that

$$
0 \in \sum_{v \in V\left(x^{*}\right)} \lambda_{\nu} s_{v}
$$

where $s_{v} \in \partial f(v)$ for all $v$, for some $\lambda$ satisfying $\lambda \geq 0, \lambda \neq 0$. Observe for $v \in \partial S$ and $s \in \partial f(v)$ that $0 \geq f(w) \geq f(v)+\langle s, w-v\rangle=\langle s, w-v\rangle$ for all $w \in S$, which implies that $s \in N_{S}(v)$, and so

$$
0 \in \sum_{v \in V\left(x^{*}\right)} \lambda_{v} s_{v}
$$

where $s_{v} \in N_{S}(v)$ for all $v$, which implies (ii).

Conversely, suppose that $\alpha^{*}=\operatorname{sym}\left(x^{*}, S\right)$, and note that for any $v \in V\left(x^{*}\right)$, $0 \notin \partial f(v)$ (otherwise $f$ would be nonnegative which contradicts Assumption A and Lemma 3). Therefore $0 \in \operatorname{conv} S V\left(x^{*}\right)$ implies that cone $S V\left(x^{*}\right)$ contains a line. Let $y \in S$ be given and define $d:=y-x^{*}$. Since $S V\left(x^{*}\right)$ contains a line, there exists $s \in S V\left(x^{*}\right)$ for which $\langle s, d\rangle \geq 0$. Let $v$ be the touching point corresponding to $s$, i.e., $v \in V\left(x^{*}\right)$ and $s \in N_{S}(v)$; then $v \in \partial S$ and $v=x^{*}-\alpha^{*}\left(w-x^{*}\right)$ for some $w \in S$ (from (4)). From (v) of Lemma 3 we have $s \in \partial f(v)$, whereby $s \in \partial f(v)$. Thus, using the subgradient inequality,

$$
\begin{aligned}
f\left(y-\alpha^{*}(w-y)\right) & =f\left(v+\left(y-\alpha^{*}(w-y)-v\right)\right) \\
\geq & f(v)+\left\langle s, y-\alpha^{*}(w-y)-v\right\rangle \\
& =\langle s, d\rangle\left(1+\alpha^{*}\right) \geq 0
\end{aligned}
$$

which shows that $y-\alpha^{*}(w-y) \notin \operatorname{int} S$. This implies that

$$
-\alpha^{*}(S-y) \nsubseteq \operatorname{int}(S-y)
$$


Then Lemma 2 implies $\operatorname{sym}(y, S) \leq \alpha^{*}$ for all $y \in S$ proving the optimality of $x^{*}$.

We close this subsection with some properties of the set of symmetry points $\mathrm{S}_{\text {opt }}(S)$. Note that $\mathrm{S}_{\text {opt }}(S)$ is not necessarily a singleton. To see how multiple symmetry points can arise, consider $S:=\left\{x \in \mathbb{R}^{3}: x_{1} \geq 0, x_{2} \geq 0, x_{1}+x_{2} \leq\right.$ $\left.1,0 \leq x_{3} \leq 1\right\}$, which is the cross product of a 2-dimensional simplex and a unit interval. Therefore $\operatorname{sym}(S)=\min \left\{\frac{1}{2}, 1\right\}=\frac{1}{2}$ and $\mathrm{S}_{\mathrm{opt}}(S)=\left\{x \in \mathbb{R}^{3}: x_{1}=x_{2}=\right.$ $\left.\frac{1}{3}, x_{3} \in\left[\frac{1}{3}, \frac{2}{3}\right]\right\}$.

Proposition 6 Under Assumption A, $S_{\text {Opt }}(S)$ is a compact convex set with no interior. If $S$ is a strictly convex set, then $S_{\text {opt }}(S)$ is a singleton.

Proof The convexity of $\mathrm{S}_{\mathrm{opt}}(S)$ follows directly from the quasiconcavity of $\operatorname{sym}(\cdot, S)$, see Theorem 1 . Let $\alpha:=\operatorname{sym}(S)$, and suppose that there exists $\hat{x} \in$ int $_{\text {Opt }}(S)$. This implies that there exists $\delta>0$ such that $\operatorname{sym}(x, S)=\alpha$ for all $x \in B(\hat{x}, \delta) \subset \mathrm{S}_{\mathrm{opt}}(S)$. Then for all $d$ satisfying $\|d\| \leq 1$ we have:

$$
\alpha(\hat{x}+\delta d-S) \subseteq S-(\hat{x}+\delta d)
$$

which implies that

$$
\alpha(\hat{x}-S)+B(0, \delta(1+\alpha)) \subseteq S-\hat{x} .
$$

Using Lemma 2, this implies $\alpha<\mathbf{s y m}(\hat{x}, S)$, which is a contradiction.

For the last statement, suppose $x_{1}, x_{2} \in \mathrm{S}_{\mathrm{opt}}(S)$ and $x_{1} \neq x_{2}$. Since any strict convex combination of elements of $S$ must lie in the interior of $S$, for any $\gamma \in(0,1)$ it follows that

$$
\left(\gamma x_{1}+(1-\gamma) x_{2}\right)-\alpha\left(S-\left(\gamma x_{1}+(1-\gamma) x_{2}\right)\right) \subseteq \text { int } S .
$$

Again using Lemma 2, it follows that $\operatorname{sym}\left(\gamma x_{1}+(1-\gamma) x_{2}, S\right)>\alpha$, which is also a contradiction.

Remark 6 In [16], Klee proved the following notable relation between $\operatorname{sym}(S)$ and the dimension of $\mathrm{S}_{\mathrm{opt}}(S)$ :

$$
\frac{1}{\operatorname{sym}(S)}+\operatorname{dim}\left(\mathrm{S}_{\text {opt }}(S)\right) \leq n,
$$

which implies that multiple symmetry points can only exist in dimensions $n \geq 3$.

\section{Computing a symmetry point of $S$ when $S$ is polyhedral}

Our interest in this section lies in computing an $\varepsilon$-approximate symmetry point of $S$, which is a point $x \in S$ that satisfies: 


$$
\operatorname{sym}(x, S) \geq(1-\varepsilon) \operatorname{sym}(S) .
$$

We focus on the polyhedral case; more specifically, we study the problem in which the convex set of interest is given by the convex hull of finitely many points or by the intersection of finitely many half-spaces.

Although the symmetry function is invariant under equivalent representations of the set $S$, the question of computing the symmetry of a point in a general convex set is not, as the following example indicates.

Example 1 Let $C^{n}=\left\{x \in \mathbb{R}^{n}:\|x\|_{\infty} \leq 1\right\}$ be the $n$-dimensional hypercube. Let $v$ be a vertex of $C^{n}$, and define $H=\left\{x \in \mathbb{R}^{n}:\langle x, v\rangle \leq n-1 / 2\right\}$, and define $S:=C^{n} \cap H$. Then $\operatorname{sym}(0, S)=1-1 / 2 n$ is obtained by considering the vertex $-v$. Assume that $S$ is given only by a membership oracle and note that $H$ cuts off a pyramid from $S$ that is completely contained in exactly one of the $2^{n}$ orthants of $\mathbb{R}^{n}$. Since we can arbitrarily choose the vertex $v$, in the worst case any deterministic algorithm will need to verify every single orthant to show that $\operatorname{sym}(0, S)<1$, leading to an exponential complexity in the dimension $n$.

This example suggests that more structure is needed for the representation of $S$ in order to compute an $\varepsilon$-approximate symmetry point of $S$. In the following two subsections we consider the cases when $S$ is given as the convex hull of finitely many points (Sect. 5.1), and as the intersection of finitely many half-spaces (Sect. 5.2).

\section{1 $S$ represented by the convex hull of points}

In this subsection we assume that $S$ is given as the convex hull of $m$ given points $w^{1}, \ldots, w^{m} \in \mathbb{R}^{n}$, i.e., $S=\operatorname{conv}\left\{w^{1}, \ldots, w^{m}\right\}$. Given $x \in S$ and a nonnegative scalar $\alpha$, it follows from (4) that $\operatorname{sym}(x, S) \geq \alpha$ if and only if

$$
(1+\alpha) x-\alpha w^{i} \in S=\operatorname{conv}\left\{w^{j}: j=1, \ldots, m\right\} \quad \text { for every } i=1, \ldots, m,
$$

which can be checked by solving a system of linear inequalities. It follows that $\operatorname{sym}(S)$ is the optimal value of the following optimization problem:

$$
\begin{aligned}
& \max _{\alpha, x, \lambda, v} \alpha \\
& \text { s.t. } \quad(1+\alpha) x-\alpha w^{i}=\sum_{k=1}^{m} \lambda_{k}^{i} w^{k}, \quad i=1, \ldots, m \\
& x=\sum_{k=1}^{m} v_{k} w^{k} \\
& e^{T} \lambda^{i}=1, \quad \lambda^{i} \geq 0, \quad i=1, \ldots, m \\
& e^{T} v=1, \quad v \geq 0 \text {, }
\end{aligned}
$$


which is almost a linear program. Note that the constraints " $x=\sum_{k=1}^{m} v_{k} w^{k}$, $e^{T} v=1, v \geq 0$ " of (31) simply state that $x$ must lie in the convex hull of the points $w^{1}, \ldots, w^{m}$. However, dividing the first set of constraints by $(1+\alpha)$ one obtains for a given $i$ :

$$
x=\left(\frac{\alpha}{1+\alpha} w^{i}+\frac{1}{1+\alpha} \sum_{k=1}^{m} \lambda_{k}^{i} w^{k}\right),
$$

which shows that these constraints themselves imply that $x$ is in the convex hull of $w^{1}, \ldots, w^{m}$, and so the former set of constraints can be eliminated. Furthermore, setting $y=(1+\alpha) x$, it follows that $\operatorname{sym}(S)$ is the optimal value of the linear program:

$$
\begin{array}{ll}
\max _{\alpha, y, \lambda} & \alpha \\
\text { s.t. } & y-\alpha w^{i}=\sum_{k=1}^{m} \lambda_{k}^{i} w^{k}, \quad i=1, \ldots, m \\
& e^{T} \lambda^{i}=1, \quad \lambda^{i} \geq 0, \quad i=1, \ldots, m,
\end{array}
$$

and that any optimal solution $\left(\alpha^{*}, y^{*}, \lambda^{*}\right)$ of (32) yields $\operatorname{sym}(S)=\alpha^{*}$ and $x^{*}=y^{*} /\left(1+\alpha^{*}\right)$ is a symmetry point of $S$.

Formulation (32) has $m^{2}$ nonnegative variables and $m n+m$ equality constraints. Moreover, the analytic center for the slice of the feasible region on the level set corresponding to $\alpha=0$ is readily available for this formulation by setting

$$
\alpha=0, \quad y=\frac{1}{m} \sum_{k=1}^{m} w^{k}, \quad \lambda^{i}=\frac{1}{m} e, i=1, \ldots, m,
$$

and therefore (32) lends itself to solution by interior-point methods so long as $m$ is not too large.

If $m$ is large it might not be attractive to solve (32) directly, and in order to develop a more attractive approach to computing $\mathbf{s y m}(S)$ we proceed as follows. Based on (31) we can compute $\operatorname{sym}(x, S)$ by simply fixing $x$. Thus, for each $i=1, \ldots, m$ define

$$
\begin{aligned}
& f_{i}(x)=\max _{\alpha_{i}, \lambda^{i}} \alpha_{i} \\
& \text { s.t. } \quad\left(1+\alpha_{i}\right) x-\alpha_{i} w^{i}=\sum_{k=1}^{m} \lambda_{k}^{i} w^{k} \\
& \\
& \quad e^{T} \lambda^{i}=1, \lambda^{i} \geq 0,
\end{aligned}
$$

and it follows that $\operatorname{sym}(x, S)=\min _{i=1, \ldots, m} f_{i}(x)$. Dividing the first constraint by $\left(1+\alpha_{i}\right)$ and defining $\theta_{i}=\frac{\alpha_{i}}{1+\alpha_{i}}$ and noting that maximizing $\theta_{i}$ is equivalent to 
maximizing $\alpha_{i}$, it follows that (33) is equivalent to:

$$
\begin{aligned}
& h_{i}(x)=\max _{\theta_{i}, \lambda^{i}} \theta_{i} \\
& \text { s.t. } \quad \theta_{i} w^{i}+\sum_{k=1}^{m} \lambda_{k}^{i} w^{k}=x \\
& \\
& e^{T} \lambda^{i}=1-\theta_{i}, \lambda^{i} \geq 0 .
\end{aligned}
$$

Now note that $h_{i}(x)$ is a concave function, whereby

$$
h(x):=\min _{i=1, \ldots, m} h_{i}(x)
$$

is also a concave function, and furthermore

$$
\frac{\operatorname{sym}(x, S)}{1+\operatorname{sym}(x, S)}=\min _{i=1, \ldots, m} h_{i}(x)=h(x) .
$$

Moreover, given a value of $x$, the computation of $h(x)$ and the computation of a subgradient of $h(\cdot)$ at $x$ is easily accomplished by solving the $m$ linear programs (34) which each have $m$ nonnegative variables and $n+1$ equality constraints. Therefore the problem of maximizing $h(x)$ is suitable for classical nondifferentiable optimization methods such as bundle methods, see [6] for example.

\section{$5.2 S$ represented by linear inequalities}

In this subsection we assume that $S$ is given as the intersection of $m$ inequalities, i.e., $S:=\left\{x \in \mathbb{R}^{n}: A x \leq b\right\}$ where $A \in \mathbb{R}^{m \times n}$ and $b \in \mathbb{R}^{m}$. We present two methods for computing an $\varepsilon$-approximate symmetry point of $S$. The first method is based on approximately solving a single linear program with $m^{2}+m$ inequalities. For such a method, an interior-point algorithm would require $O\left(\mathrm{~m}^{6}\right)$ operations per Newton step, which is clearly unattractive. Our second method involves solving $m+1$ linear programs each of which involves $m$ linear inequalities in $n$ unrestricted variables. This method is more complicated to evaluate, but is clearly more attractive should one want to compute an $\varepsilon$-approximate symmetry point in practice.

Let $\bar{x} \in S$ be given, and let $\alpha \leq \operatorname{sym}(\bar{x}, S)$. Then from the definition of $\operatorname{sym}(\cdot, S)$ in (1) we have:

$$
A(\bar{x}+v) \leq b \Rightarrow A(\bar{x}-\alpha v) \leq b,
$$

which we restate as:

$$
A v \leq b-A \bar{x} \Rightarrow-\alpha A_{i} \cdot v \leq b_{i}-A_{i} \cdot \bar{x}, \quad i=1, \ldots, m
$$


Now apply a theorem of the alternative to each of the $i=1, \ldots, m$ implications (35). Then (35) is true if and only if there exists an $m \times m$ matrix $\Lambda$ of multipliers that satisfies:

$$
\begin{gathered}
\Lambda A=-\alpha A \\
\Lambda(b-A \bar{x}) \leq b-A \bar{x} \\
\Lambda \geq 0 .
\end{gathered}
$$

Here " $\Lambda \geq 0$ " is componentwise for all $m^{2}$ components of $\Lambda$. This means that $\operatorname{sym}(\bar{x}, S) \geq \alpha$ if and only if (36) has a feasible solution. This also implies that $\operatorname{sym}(S)$ is the optimal objective value of the following optimization problem:

$$
\begin{array}{ll}
\max _{x, \Lambda, \alpha} \alpha & \\
\text { s.t. } & \Lambda A=-\alpha A \\
& \Lambda(b-A x) \leq b-A x \\
& \Lambda \geq 0,
\end{array}
$$

and any solution $\left(x^{*}, \Lambda^{*}, \alpha^{*}\right)$ of (37) satisfies $\operatorname{sym}\left(x^{*}, S\right)=\alpha^{*}$. Notice that (37) is not a linear program. To convert it to a linear program, we make the following change of variables:

$$
\gamma=\frac{1}{\alpha}, \quad \Pi=\frac{1}{\alpha} \Lambda, \quad y=\frac{1+\alpha}{\alpha} x,
$$

which can be used to transform (37) to the following linear program:

$$
\begin{array}{ll}
\min _{y, \Pi, \gamma} \gamma & \\
\text { s.t. } & \Pi A=-A \\
& \Pi b+A y-b \gamma \leq 0 \\
& \Pi \geq 0 .
\end{array}
$$

If $\left(y^{*}, \Pi^{*}, \gamma^{*}\right)$ is a solution of (38), then $\alpha^{*}:=1 / \gamma^{*}=\mathbf{s y m}(S)$ and $x^{*}:=\frac{1}{1+\gamma^{*}} y^{*} \in \mathrm{S}_{\mathrm{Opt}}(S)$. Notice that (38) has $m^{2}+m$ inequalities and $m n$ equations. Suppose we know an approximate analytic center $x^{a}$ of $S$. Then it is possible to develop an interior-point method approach to solving (38) using information from $x^{a}$, and one can prove that a suitable interior-point method will compute an $\varepsilon$-approximate symmetry point of $S$ in $O\left(m \ln \left(\frac{m}{\varepsilon}\right)\right)$ iterations of Newton's method. However, due to the $m^{2}+m$ inequalities, each Newton step requires $O\left(\mathrm{~m}^{6}\right)$ operations, which is clearly unattractive.

In order to improve on the previous approach, we define the following scalar quantities $\delta_{i}^{*}, i=1, \ldots, m$ :

$$
\begin{aligned}
\delta_{i}^{*}:=\max _{x}-A_{i} \cdot x \\
\text { s.t. } \quad A x \leq b,
\end{aligned}
$$

and notice that $b_{i}+\delta_{i}^{*}$ is the range of $A_{i} x$ over $x \in S$ unless the $i$ th constraint is never active. We compute $\delta_{i}^{*}, i=1, \ldots, m$ by solving $m$ linear programs whose 
feasible region is exactly $S$. It then follows directly from Proposition 1 that

$$
\operatorname{sym}(x, S)=\min _{i=1, \ldots, m}\left\{\frac{b_{i}-A_{i} \cdot x}{\delta_{i}^{*}+A_{i} \cdot x}\right\} .
$$

We now use (40) to create another single linear program to compute $\operatorname{sym}(S)$ as follows. Let $\delta^{*}:=\left(\delta_{1}^{*}, \ldots, \delta_{m}^{*}\right)$ and consider the following linear program that uses $\delta^{*}$ in the data:

$$
\begin{array}{cl}
\max _{x, \check{\theta}} & \check{\theta} \\
\text { s.t. } & A x+\check{\theta}\left(\delta^{*}+b\right) \leq b .
\end{array}
$$

Proposition 7 Let $\left(x^{*}, \check{\theta}^{*}\right)$ be an optimal solution of the linear program (41). Then $x^{*}$ is a symmetry point of $S$ and $\mathbf{s y m}(S)=\frac{\breve{\theta}^{*}}{1-\check{\theta}^{*}}$.

Proof Suppose that $(x, \check{\theta})$ is a feasible solution of (41). Then $\frac{1}{\check{\theta}} \geq \frac{\delta_{i}^{*}+b_{i}}{b_{i}-A_{i} \cdot x}$, whereby

$$
\frac{1-\check{\theta}}{\check{\theta}}=\frac{1}{\check{\theta}}-1 \geq \frac{\delta_{i}^{*}+A_{i \cdot} x}{b_{i}-A_{i \cdot} \cdot x}
$$

and so

$$
\frac{b_{i}-A_{i \cdot} \cdot x}{\delta_{i}^{*}+A_{i} \cdot x} \geq \frac{\check{\theta}}{1-\check{\theta}}, \quad i=1, \ldots, m .
$$

It then follows from Proposition 1 that $\operatorname{sym}(x, S) \geq \frac{\check{\theta}}{1-\check{\theta}}$, which implies that $\operatorname{sym}(S) \geq \frac{\check{\theta}^{*}}{1-\breve{\theta}^{*}}$. The proof of the reverse inequality follows similarly.

This yields the following "exact" method for computing $\operatorname{sym}(S)$ and a symmetry point $x^{*}$ :

\section{Exact method:}

Step 1 For $i=1, \ldots, m$ solve the linear program (39) for $\delta_{i}^{*}$.

Step 2 Let $\delta^{*}:=\left(\delta_{1}^{*}, \ldots, \delta_{m}^{*}\right)$. Solve the linear program (41) for an optimal solution $\left(x^{*}, \check{\theta}^{*}\right)$. Then $x^{*} \in \mathrm{S}_{\mathrm{Opt}}(S)$ and $\operatorname{sym}(S)=\frac{\check{\theta}^{*}}{1-\check{\theta}^{*}}$.

This method involves the exact solution of $m+1$ linear programs. The first $m$ linear programs can actually be solved in parallel, and their optimal objective values are used in the data for the $(m+1)$ st linear program. The first $m$ linear programs each have $m$ inequalities in $n$ unrestricted unknowns. The last linear program has $m$ inequalities and $n+1$ unrestricted unknowns, and could be reduced to $n$ unknowns using variable elimination if so desired. 
Remark 7 Although $\mathbf{s y m}(S)$ can be computed via linear programming when $S$ is represented either as a convex hull of points or as the intersection of halfspaces, the latter case appears to be genuinely easier; indeed, the Exact Method solves a sequence of $m+1$ linear programs of size $m \times n$ when $S$ is given by half-spaces, instead of a single linear program with $m^{2}$ inequalities when $S$ is represented as the convex hull of points. It is an open question whether there is a more efficient scheme than solving (32) for computing $\operatorname{sym}(S)$ when $S$ is represented as the convex hull of points.

From a complexity perspective, it is desirable to consider solving the $m+1$ linear programs of the Exact Method for a feasible and near-optimal solution. Ordinarily, this would be easy to analyze. But in this case, the approximately optimal solution to the $m$ linear programs (39) will then yield imprecise input data for the linear program (41). Nevertheless, one can construct an inexact method with an appropriately good complexity bound. Below is a description of such a method.

\section{Inexact method:}

Step 1 For $i=1, \ldots, m$, approximately solve the linear program (39), stopping each linear program when a feasible solution $\bar{x}$ is computed for which the duality gap $\bar{g}$ satisfies $\bar{g} \leq \frac{\varepsilon\left(b_{i}-A_{i} \cdot \bar{x}\right)}{4.1}$. Set $\bar{\delta}_{i} \leftarrow-A_{i} \cdot \bar{x}$.

Step 2 Let $\bar{\delta}:=\left(\bar{\delta}_{1}, \ldots, \bar{\delta}_{m}\right)$. Approximately solve the linear program

$$
\begin{array}{ll}
\max _{x, \theta} & \theta \\
\text { s.t. } & A x+\theta(\bar{\delta}+b) \leq b,
\end{array}
$$

stopping when a feasible solution $(\bar{x}, \bar{\theta})$ is computed for which the duality gap $\bar{g}$ satisfies $\bar{\theta} \geq(\bar{\theta}+\bar{g})\left(1-\frac{\varepsilon}{4.1}\right)$. Then $\bar{x}$ is an $\varepsilon$-approximate symmetry point of $S$ and $\frac{\bar{\theta}}{1-\bar{\theta}}(1-\varepsilon / 2) \leq \mathbf{s y m}(S) \leq \frac{\bar{\theta}}{1-\bar{\theta}}(1+2 \varepsilon / 3)$.

Notice that this method requires that the LP solver computes primal and dual feasible points (or simply primal feasible points and the duality gap) at each of its iterations; such a requirement is satisfied, for example, by a standard feasible interior-point method, see Appendix B.

In order to prove a complexity bound for the Inexact Method, we will assume that $S$ is bounded and has an interior, and that an approximate analytic center $x^{a}$ of the system $A x \leq b$ has already been computed; for details also see Appendix B.

Theorem 13 Let $\varepsilon \in(0,1 / 10)$ be given. Suppose that $n \geq 2$ and $x^{a}$ is a $\beta=\frac{1}{8}$ approximate analytic center of $S$. Then starting with $x^{a}$ and using a standard feasible interior-point method to solve each of the linear programs in Steps 1 and 2 , the inexact method will compute an $\varepsilon$-approximate symmetry point of $S$ in no 
more than

$$
\left\lceil 10 m^{1.5} \ln \left(\frac{10 m}{\varepsilon}\right)\right\rceil
$$

total iterations of Newton's method.

The following proposition validates the assertions made at the end of Step 2 of the Inexact Method.

Proposition 8 Let $\varepsilon \in(0,1 / 10)$ be given, set $\tilde{\varepsilon}:=\varepsilon / 4.1$, and suppose that Steps 1 and 2 of the inexact method are executed, with output $(\bar{x}, \bar{\theta})$. Then

(i) $\bar{\delta}=\left(\bar{\delta}_{1}, \ldots, \bar{\delta}_{m}\right)$ satisfies $(1-\tilde{\varepsilon})\left(b_{i}+\delta_{i}^{*}\right) \leq\left(b_{i}+\bar{\delta}_{i}\right) \leq\left(b_{i}+\delta_{i}^{*}\right)$ for $i=1, \ldots, m$.

(ii) For any given $x \in S, \theta:=\min _{i}\left\{\frac{b_{i}-A_{i} \cdot x}{\bar{\delta}_{i}+b_{i}}\right\}$ satisfies

$$
\operatorname{sym}(x, S) \in\left[\frac{\theta}{1-\theta}\left(1-\frac{2 \tilde{\varepsilon}}{1-\tilde{\varepsilon}}\right), \frac{\theta}{1-\theta}\right],
$$

(iii) $\operatorname{sym}(\bar{x}, S) \geq(1-\varepsilon) \mathbf{s y m}(S)$, and

(iv) $\frac{\bar{\theta}}{1-\bar{\theta}}(1-\varepsilon / 2) \leq \mathbf{s y m}(S) \leq \frac{\bar{\theta}}{1-\bar{\theta}}(1+2 \varepsilon / 3)$.

Proof For a given $i=1, \ldots, m$ let $\bar{g}$ denote the duality gap computed in the stopping criterion of Step 1 of the inexact method. Then $\delta_{i}^{*} \geq \bar{\delta}_{i} \geq \delta_{i}^{*}-\bar{g} \geq$ $\delta_{i}^{*}-\tilde{\varepsilon}\left(b_{i}-A_{i} \cdot \bar{x}\right) \geq \delta_{i}^{*}-\tilde{\varepsilon}\left(b_{i}+\delta_{i}^{*}\right)$, which implies

$$
(1-\tilde{\varepsilon})\left(b_{i}+\delta_{i}^{*}\right) \leq\left(b_{i}+\bar{\delta}_{i}\right) \leq\left(b_{i}+\delta_{i}^{*}\right),
$$

thus proving (i). To prove (ii), let $x \in S$ be given and let $\alpha:=\operatorname{sym}(x, S)$ and $\check{\theta}:=\min _{i}\left\{\frac{b_{i}-A_{i} \cdot x}{\delta_{i}^{*}+b_{i}}\right\}$. Then from Proposition 1 we have

$$
\alpha=\min _{i}\left\{\frac{b_{i}-A_{i \cdot} x}{\delta_{i}^{*}+A_{i \cdot} \cdot x}\right\}=\frac{\check{\theta}}{1-\check{\theta}} .
$$

Notice that $\bar{\delta}_{i} \leq \delta_{i}^{*}$ for all $i$, whereby $\theta \geq \check{\theta}$, which implies that $\alpha=\frac{\check{\theta}}{1-\check{\theta}} \leq \frac{\theta}{1-\theta}$. We also see from (43) that $\check{\theta} \leq 1 / 2$. Next notice that (i) implies that

$$
1 / 2 \geq \check{\theta} \geq \theta(1-\tilde{\varepsilon})
$$


Therefore

$$
\begin{aligned}
\alpha=\frac{\check{\theta}}{1-\check{\theta}} & \geq \frac{\theta(1-\tilde{\varepsilon})}{1-\check{\theta}}=\frac{\theta(1-\tilde{\varepsilon})}{1-\theta} \frac{1-\theta}{1-\check{\theta}} \\
& =\frac{\theta(1-\tilde{\varepsilon})}{1-\theta}\left(1+\frac{\check{\theta}-\theta}{1-\check{\theta}}\right) \geq \frac{\theta(1-\tilde{\varepsilon})}{1-\theta}\left(1+\frac{\left.\check{\theta}-\frac{1}{1-\tilde{\varepsilon}}\right)}{1-\check{\theta}}\right) \\
& =\frac{\theta(1-\tilde{\varepsilon})}{1-\theta}\left(1+\frac{\tilde{\theta}\left(\frac{-\tilde{\varepsilon}}{1-\tilde{\varepsilon}}\right)}{1-\check{\theta}}\right) \geq \frac{\theta(1-\tilde{\varepsilon})}{1-\theta}\left(1-\frac{\tilde{\varepsilon}}{1-\tilde{\varepsilon}}\right) \\
& \geq \frac{\theta}{1-\theta}\left(1-\frac{2 \tilde{\varepsilon}}{1-\tilde{\varepsilon}}\right)
\end{aligned}
$$

where the next-to-last inequality follows from $\check{\theta} \in[0,1 / 2]$, thereby showing (ii).

Let $\theta^{*}$ denote the optimal objective value of (42), and notice that $\bar{\delta} \leq \delta^{*}$ implies that $\theta^{*} \geq \check{\theta}^{*}$. Now let $\bar{g}$ be the duality gap computed when the stopping criterion in Step 2 is met. Then

$$
\theta \geq \bar{\theta} \geq(\bar{\theta}+\bar{g})(1-\tilde{\varepsilon}) \geq \theta^{*}(1-\tilde{\varepsilon}) \geq \check{\theta}^{*}(1-\tilde{\varepsilon})
$$

From (ii) and (46) we have

$$
\begin{aligned}
\operatorname{sym}(\bar{x}, S) & \geq \frac{\theta}{1-\theta}\left(1-\frac{2 \tilde{\varepsilon}}{1-\tilde{\varepsilon}}\right) \geq \frac{\check{\theta}^{*}(1-\tilde{\varepsilon})}{1-\check{\theta}^{*}(1-\tilde{\varepsilon})}\left(1-\frac{2 \tilde{\varepsilon}}{1-\tilde{\varepsilon}}\right) \\
& =\frac{\check{\theta}^{*}(1-\tilde{\varepsilon})}{1-\check{\theta}^{*}}\left(1-\frac{2 \tilde{\varepsilon}}{1-\tilde{\varepsilon}}\right) \frac{1-\check{\theta}^{*}}{1-\check{\theta}^{*}(1-\tilde{\varepsilon})} \\
& \geq \operatorname{sym}(S)(1-\tilde{\varepsilon})\left(1-\frac{2 \tilde{\varepsilon}}{1-\tilde{\varepsilon}}\right)\left(\frac{1 / 2}{1-1 / 2+(1 / 2) \tilde{\varepsilon}}\right) \\
& =\operatorname{sym}(S)(1-\tilde{\varepsilon})\left(1-\frac{2 \tilde{\varepsilon}}{1-\tilde{\varepsilon}}\right)\left(1-\frac{\tilde{\varepsilon}}{1+\tilde{\varepsilon}}\right) \\
& \geq \operatorname{sym}(S)\left(1-\frac{4 \tilde{\varepsilon}}{1-\tilde{\varepsilon}}\right) \geq \mathbf{s y m}(S)(1-\varepsilon),
\end{aligned}
$$

where the middle inequality uses the fact that $\check{\theta}^{*} \in[0,1 / 2]$, and the final inequality uses the fact that $\epsilon \in(0,1 / 10]$, thus showing (iii).

To prove (iv), note that

$$
\operatorname{sym}(S) \geq \operatorname{sym}(\bar{x}, S) \geq \frac{\theta}{1-\theta}\left(1-\frac{2 \tilde{\varepsilon}}{1-\tilde{\varepsilon}}\right) \geq \frac{\theta}{1-\theta}\left(1-\frac{\varepsilon}{2}\right) \geq \frac{\bar{\theta}}{1-\bar{\theta}}\left(1-\frac{\varepsilon}{2}\right)
$$


where the second inequality follows from part (ii), the third inequality follows since $\varepsilon \leq 1 / 10$, and the fourth inequality uses $\bar{\theta} \leq \theta$. Last of all, we have

$$
\begin{aligned}
\operatorname{sym}(S) & =\frac{\check{\theta}^{*}}{1-\check{\theta}^{*}} \leq \frac{\frac{\bar{\theta}}{1-\tilde{\varepsilon}}}{1-\frac{\bar{\theta}}{1-\tilde{\varepsilon}}}=\frac{\bar{\theta}}{1-\bar{\theta}-\tilde{\varepsilon}}=\frac{\bar{\theta}}{1-\bar{\theta}}\left(\frac{1-\bar{\theta}}{1-\bar{\theta}-\tilde{\varepsilon}}\right) \\
& \leq \frac{\bar{\theta}}{1-\bar{\theta}}\left(1+\frac{2 \varepsilon}{3}\right),
\end{aligned}
$$

where the first equality is from Proposition 7 , the first inequality follows from (46), and the last inequality follows since $\varepsilon \leq 1 / 10$ and (44) implies that $\bar{\theta} \leq$ $\theta \leq 41 / 80$.

It remains to prove the complexity bound of Theorem 13, which will be accomplished with the help of the following two propositions.

Proposition 9 Let $\varepsilon \in(0,1 / 10)$ be given, and set $\tilde{\varepsilon}:=\varepsilon / 4.1$. Suppose that $x^{a}$ is a $\beta=\frac{1}{8}$-approximate analytic center of $S$. Then starting with $x^{a}$, the stopping criterion of each linear program in Step 1 will be reached in no more than

$$
\left\lceil(2+4 \sqrt{m}) \ln \left(\frac{42 m}{\varepsilon}\right)\right\rceil
$$

iterations of Newton's method.

Proof Step 1 is used to approximately solve each of the linear programs (39) for $i=1, \ldots, m$. Let us fix a given $i$, and define $\lambda:=-e^{i}$ where $e^{i}$ is the $i^{\text {th }}$ unit vector in $\mathbb{R}^{m}$. Then from Theorem 14 with $(M, f, c)=\left(A, b,-A_{i}\right.$. ) we can bound the iterations used to solve (39) by

$$
\left\lceil(2+4 \sqrt{m}) \ln \left(\frac{10 m\left\|S^{a} \lambda\right\|}{\bar{g}}\right)^{+}\right\rceil .
$$

Now notice that $\left\|S^{a} \lambda\right\|=s_{i}^{a}$. Let $(\bar{x}, \bar{s})$ denote the primal solution and slack vector computed in Step 1 when the stopping criterion is met. Also, to keep the analysis simple, we assume that the stopping criterion is met exactly. We have:

$$
\bar{s}_{i}=b_{i}-A_{i \cdot} \bar{x} \geq b_{i}+\delta_{i}^{*}-\bar{g} \geq b_{i}+\delta_{i}^{*}-\tilde{\varepsilon} \bar{s}_{i} \geq b_{i}-A_{i \cdot} x^{a}-\tilde{\varepsilon} \bar{s}_{i}=s_{i}^{a}-\tilde{\varepsilon} \bar{s}_{i},
$$

whereby $s_{i}^{a} \leq \bar{s}_{i}(1+\tilde{\varepsilon})$. Therefore

$$
\frac{10 m\left\|S^{a} \lambda\right\|}{\bar{g}}=\frac{10 m s_{i}^{a}}{\tilde{\varepsilon} \bar{s}_{i}} \leq \frac{10 m(1+\tilde{\varepsilon})}{\tilde{\varepsilon}}=\frac{41 m(1+\varepsilon / 4.1)}{\varepsilon} \leq \frac{42 m}{\varepsilon},
$$

since in particular $\varepsilon \in(0,1 / 10)$. Substituting this inequality into (48) completes the proof. 
Proposition 10 Let $\varepsilon \in(0,1 / 10)$ be given, $m \geq 3$ and set $\tilde{\varepsilon}:=\varepsilon / 4$.1. Suppose that $x^{a}$ is a $\beta=\frac{1}{8}$-approximate analytic center of $S$. Then starting with $x^{a}$, the stopping criterion of the linear program in Step 2 will be reached in no more than

$$
\left\lceil(2+4 \sqrt{m}) \ln \left(\frac{6 m}{\varepsilon}\right)\right\rceil
$$

iterations of Newton's method.

Proof Let $s^{a}=b-A x^{a}$ and let $z^{a}$ denote the dual multipliers associated with (52) for $M=A$ and $f=b$. It follows from (52) and $m \geq 3$ that

$$
\left(s^{a}\right)^{T} z^{a}=e^{T}\left(S^{a} z^{a}-e+e\right) \geq-\frac{1}{8} \sqrt{m}+m \geq \frac{9 m}{10} .
$$

Setting $(M, f, d)=(A, b,(\bar{\delta}+b))$ we see that (42) is an instance of (53), and from Theorem 15 we can bound the iterations used to solve (42) by

$$
\left\lceil(2+4 \sqrt{m}) \ln \left(\frac{1.25 m}{\bar{g} \cdot(\bar{\delta}+b)^{T} z^{a}}\right)^{+}\right\rceil .
$$

We have

$$
(\bar{\delta}+b)^{T} z^{a} \geq\left(b+\delta^{*}\right)^{T} z^{a}(1-\tilde{\varepsilon}) \geq\left(s^{a}\right)^{T} z^{a}(1-\tilde{\varepsilon}) \geq \frac{9 m(1-\tilde{\varepsilon})}{10}
$$

where the first inequality follows from part (i) of Proposition 8 , the second inequality follows from $b+\delta^{*} \geq b-A x^{a}=s^{a}$, and the third inequality follows from (49). We next bound $\bar{g}$. To keep things simple we again assume that the stopping criterion in Step 2 is satisfied exactly, whereby

$$
\frac{1}{\bar{g}}=\frac{1-\tilde{\varepsilon}}{\tilde{\varepsilon}} \frac{1}{\bar{\theta}} \leq \frac{1}{\tilde{\varepsilon} \cdot \check{\theta}^{*}}=\frac{4.1}{\varepsilon}\left(1+\frac{1}{\operatorname{sym}(S)}\right) \leq \frac{4.1}{\varepsilon}(1+n) \leq \frac{4.1 m}{\varepsilon} .
$$

Here the first inequality follows from (46), the second equality follows from Proposition 7, the second inequality follows from Remark 3, and the last inequality follows since $S$ is assumed to be bounded and so $m \geq n+1$. Combining the bounds on $(\bar{\delta}+b)^{T} z^{a}$ and $\bar{g}$ we then bound the logarithm term in the statement of the proposition as follows:

$$
\frac{1.25 m}{\bar{g} \cdot(\bar{\delta}+b)^{T} z^{a}} \leq \frac{1.25 m \cdot 4.1 m \cdot 10}{9 m \varepsilon(1-\tilde{\varepsilon})} \leq \frac{6 m}{\varepsilon},
$$

since $\varepsilon \in(0,1 / 10)$ implies that $\tilde{\varepsilon} \leq 1 / 41$. This completes the proof. 
Proof of complexity bound of Theorem 13 From Propositions 9 and 10 it follows that the total number of Newton steps computed by the Inexact Method is bounded from above by:

$$
m\left\lceil(2+4 \sqrt{m}) \ln \left(\frac{42 m}{\varepsilon}\right)\right\rceil+\left\lceil(2+4 \sqrt{m}) \ln \left(\frac{6 m}{\varepsilon}\right)\right\rceil \leq\left\lceil 10 m^{1.5} \ln \left(\frac{10 m}{\varepsilon}\right)\right\rceil
$$

since $m \geq n+1 \geq 3$ and $\varepsilon<1 / 10$.

Acknowledgments We thank Arkadi Nemirovski for his insightful comments on the subject of symmetry, and for contributing the half-ball example which we used at the end of Sect. 3.2. We also thank the referees for their comments which have improved the exposition and organization of the paper.

\section{Appendix}

\section{$A \operatorname{sym}(x, S)$ and $\operatorname{sym}(S)$ under relaxed assumptions}

All of the results in this paper are based on Assumption A, namely that $S$ is a closed, bounded, convex set with an interior. Herein we discuss the implications of relaxing this set of assumptions.

As mentioned earlier, the assumption that $S$ has an interior is a matter of convenience, as we could instead work with the relative interior of $S$ on the affine hull of $S$, at considerable notational and expository expense.

The assumption that $S$ is closed is also a matter of convenience, as most of the statements contained in the body of the paper would still remain valid by replacing inf $\leftarrow$ min and sup $\leftarrow$ max and/or by working with the closure of $S$, etc.

Suppose that we relax the assumption that $S$ is bounded. If $S$ is unbounded then $S$ has a non-empty recession cone. In the case when the recession cone of $S$ is not a subspace, then $\operatorname{sym}(S)=0$. However, the case when the recession is a subspace is a bit more interesting:

Lemma 4 Suppose that $S=P+H$, where $H$ is a subspace and $P$ is a bounded convex set in $H^{\perp}$, and $x \in S$; then $\operatorname{sym}(x, S)$ is completely defined by $P$, i.e., $\operatorname{sym}(x, S)=\operatorname{sym}(w, P)$ where $x=w+h$ and $(w, h) \in H^{\perp} \times H$.

Proof Without loss of generality, we can assume that $x=0$ since symmetry is invariant under translation. Trivially, $-\alpha S \subseteq S$ if and only if $-\alpha(P+H) \subseteq$ $(P+H)$. Since $P$ and $H$ lie in orthogonal spaces, for each $x \in S$, there exist a unique $(w, h) \in P \times H$ such that $x=w+h$. Since $-\alpha H=H,-\alpha x \in S$ if and only if $-\alpha w \in P$. 


\section{B Standard interior-point method for linear programming}

Consider the following linear programming problem in "dual" form, where $M$ is an $m \times k$ matrix:

$$
\begin{aligned}
& P: \quad \mathrm{VAL}:=\max _{x, s} \quad c^{T} x \\
& \text { s.t. } \quad M x+s=f \\
& s \geq 0 \\
& x \in \mathbb{R}^{k}, \quad s \in \mathbb{R}^{m} .
\end{aligned}
$$

For $\beta \in(0,1)$, a $\beta$-approximate analytic center of the primal feasibility inequalities $M x \leq f$ is a feasible solution $x^{a}$ of $P$ (together with its slack vector $s^{a}=$ $\left.f-M x^{a}\right)$ for which there exists dual multipliers $z^{a}$ that satisfy:

$$
\begin{aligned}
& M x^{a}+s^{a}=f, s^{a}>0 \\
& M^{T} z^{a}=0 \\
& \left\|S^{a} z^{a}-e\right\| \leq \beta
\end{aligned}
$$

where $S$ is the diagonal matrix whose diagonal entries correspond to the components of $s$. Following [20] or [21], one can prove the following result about the efficiency of a standard primal interior-point method for approximately solving $P$.

Theorem 14 Suppose that $\beta=1 / 8$ and that $\left(x^{a}, s^{a}, z^{a}\right)$ is a given $\beta$-approximate analytic center of the feasibility inequalities of $P$, and that $c=M^{T} \lambda$ for some $\lambda \in \mathbb{R}^{m}$. Then $\left(x^{a}, s^{a}, z^{a}\right)$ can be used to start a standard interior-point method that will compute a feasible solution of $P$ with duality gap at most $\bar{g}$ in at most

$$
\left\lceil(2+4 \sqrt{m}) \ln \left(\frac{10 m\left\|S^{a} \lambda\right\|}{\bar{g}}\right)^{+}\right\rceil
$$

iterations of Newton method.

Now consider the following linear programming problem format:

$$
\begin{aligned}
P^{\prime}: \quad \mathrm{VAL}:=\max _{x, \theta} \quad & \theta \\
\text { s.t. } \quad & M x+d \theta+s=f \\
& s \geq 0 \\
& x \in \mathbb{R}^{k}, \quad \theta \in \mathbb{R}, \quad s \in \mathbb{R}^{m} .
\end{aligned}
$$

Again following [20] or [21], one can prove the following result about the efficiency of a standard primal interior-point method for approximately solving $P$.

Theorem 15 Suppose that $\beta=1 / 8$ and that $\left(x^{a}, s^{a}, z^{a}\right)$ is a given $\beta$-approximate analytic center of the feasibility inequalities of $P$, and that $d^{T} z^{a}>0$. Then 
$\left(x^{a}, s^{a}, z^{a}\right)$ can be used to start a standard interior-point method that will compute a feasible solution of $P^{\prime}$ with duality gap at most $\bar{g}$ in at most

$$
\left\lceil(2+4 \sqrt{m}) \ln \left(\frac{1.25 m}{\bar{g} \cdot\left(d^{T} z^{a}\right)}\right)^{+}\right\rceil
$$

iterations of Newton method.

\section{References}

1. Anderson, T.W.: The integral of a symmetric unimodal function. Proc. Amer. Math. Soc. 6,170$176(1955)$

2. Ball, K.: An elementary introduction to modern convex geometry. Flavors of Geometry, MSRI Publications, Vol. 31 (1997)

3. Barnes, E.: Private communication (1998)

4. Belloni, A., Freund, R.M.: Projective pre-conditioners for improving the behavior of a homogeneous conic linear system. MIT ORC Working Paper OR-375-05 May 2005

5. Bertsimas, D., Vempala, S.: Solving convex programs by random walks. J. ACM 51(4), 540556 (2004)

6. Bonnans, J.F., Gilbert, J.C., Lemaréchal, C., Sagastizábal, C.: Numerical Optimization: Theoretical and Practical Aspects. p. xiv+423. Universitext, Springer, Berlin Heidelbreg New York (2003)

7. Dudley, R.: Uniform Central Limit Thoerems. Cambridge University Press (2000)

8. Eaves, B.C., Freund, R.M.: Optimal scaling of balls and polyhedra. Math. Program. 23, 138147 (1982)

9. Epelman, M., Freund, R.M.: A new condition measure, preconditioners, and relations between different measures of conditioning for conic linear systems. SIAM J. Optim. 12(3), 627655 (2002)

10. Gardner, R.J.: The Brunn-Minkowski inequality. Bull. Am. Math. Soc. 39(3), 355-405 (2002)

11. Grünbaum, B.: Partitions of mass-distributions and convex bodies by hyperplanes. Pacific J. Math. 10, 1257-1261 (1960)

12. Grünbaum, B.: Measures of symmetry for convex sets. In: Convexity, Proceedings of Symposia in Pure Mathematics vol. 7, pp. 233-270 American Mathematical Society, Providence (1963)

13. Grötschel, M., Lovász, L., Schrijver, A.: Geometric Algorithms and Combinatorial Optimization. Springer, Berlin Heidelberg New York (1994)

14. Hammer, P.C.: The centroid of a convex body. Proc. Amer. Math. Soc. 5, 522-525 (1951)

15. John, F.: Extremum problems with inequalities as subsidiary conditions. In: Studies and Essays, pp. 187-204. Presented to R. Courant on His 60th Birthday, Interscience, New York (1948)

16. Klee, V.: The critical set of convex body. Am. J. Math. 75, 178-188 (1953)

17. Lovász, L., Vempala, S.: Logconcave functions: Geometry and efficient sampling algorithms. In: Proc. of the 44th IEEE Foundations of Computer Science, Boston (2003)

18. Minkowski, H.: Allegemeine Lehzätze über konvexe Polyeder. Ges. Abh. 2, 103-121 (1911)

19. Renegar, J.: A Mathematical View of Interior-Point Methods in Convex Optimization. MPSSIAM Series on Optimization (2001)

20. Roos, C., Terlaky, T., Vial, J.-P.: Theory and Algorithms for Linear Optimization: An Interior Point Approach. Wiley, New York (1997)

21. Tseng, P.: Complexity analysis of a linear complementarity algorithm based on a Lyapunov function. Math. Program. 53, 297-306 (1992) 